\title{
PKA signaling drives reticularis differentiation and sexually dimorphic adrenal cortex renewal
}

\author{
Typhanie Dumontet, ${ }^{1}$ Isabelle Sahut-Barnola, ${ }^{1}$ Amandine Septier, ${ }^{1}$ Nathanaëlle Montanier, ${ }^{1}$ \\ Ingrid Plotton, ${ }^{2}$ Florence Roucher-Boulez, ${ }^{2}$ Véronique Ducros, ${ }^{3}$ Anne-Marie Lefrançois-Martinez, ${ }^{1}$ \\ Jean-Christophe Pointud, ${ }^{1}$ Mohamad Zubair, ${ }^{4}$ Ken-Ichirou Morohashi, ${ }^{4}$ David T. Breault,, ${ }^{5,6}$ \\ Pierre Val, ${ }^{1}$ and Antoine Martinez ${ }^{1}$ \\ 'CReD, Université Clermont Auvergne, CNRS, INSERM, Clermont-Ferrand, France. ${ }^{2}$ Molecular Endocrinology and Rare \\ Diseases, University Hospital, Claude Bernard Lyon 1 University, Bron, France. ${ }^{3}$ Unit of Hormone and Nutrition, \\ Department of Biochemistry, Toxicology and Pharmacology, University Hospital, Grenoble, France. ${ }^{4}$ Department of \\ Molecular Biology, Graduate School of Medical Sciences, Kyushu University, Fukuoka, Japan. ${ }^{5}$ Division of Endocrinology, \\ Boston Children's Hospital, Department of Pediatrics, Harvard Medical School, Boston, Massachusetts, USA. ${ }^{6} \mathrm{Harvard}$ \\ Stem Cell Institute, Cambridge, Massachusetts, USA
}

The adrenal cortex undergoes remodeling during fetal and postnatal life. How zona reticularis emerges in the postnatal gland to support adrenarche, a process whereby higher primates increase prepubertal androgen secretion, is unknown. Using cell-fate mapping and gene deletion studies in mice, we show that activation of PKA has no effect on the fetal cortex, while it accelerates regeneration of the adult cortex, triggers zona fasciculata differentiation that is subsequently converted into a functional reticularis-like zone, and drives hypersecretion syndromes. Remarkably, PKA effects are influenced by sex. Indeed, testicular androgens increase WNT signaling that antagonizes PKA, leading to slower adrenocortical cell turnover and delayed phenotype whereas gonadectomy sensitizes males to hypercorticism and reticularis-like formation. Thus, reticularis results from ultimate centripetal conversion of adult cortex under the combined effects of PKA and cell turnover that dictate organ size. We show that PKA-induced progenitor recruitment is sexually dimorphic and may provide a paradigm for overrepresentation of women in adrenal diseases.

Conflict of interest: The authors have declared that no conflict of interest exists.

Submitted: October 31, 2017 Accepted: December 19, 2017 Published: January 25, 2018

\section{Reference information:} JCI Insight. 2018;3(2):e98394. https://doi.org/10.1172/jci. insight. 98394.

\section{Introduction}

The human fetal and adult adrenocortical glands produce corticosteroid hormones essential for pregnancy and body homeostasis. The adult adrenocortical gland is divided into three histologic and functional layers of steroidogenic cells, each expressing specific sets of enzymes. This results in the production of mineralocorticoids in the outer zona glomerulosa $(z G)$, glucocorticoids in the intermediate zona fasciculata (zF), and androgens in the inner zona reticularis (zR). Whereas mineralocorticoid synthesis is controlled by angiotensin II and $\mathrm{K}^{+}$levels, both glucocorticoid and adrenal androgen synthesis are under the control of pituitary adrenocorticotropic hormone (ACTH). The human adrenal cortex undergoes deep remodeling during the perinatal period and postnatal life. From the 10th week of gestation, the fetal adrenal cortex is divided into two distinct zones, the outer definitive zone, which remains minimally active until the end of the second trimester, and a large inner fetal zone. The fetal zone expresses CYP17, an enzyme that enables the synthesis of large amounts of dehydroepiandrosterone (DHEA)/DHEA sulfate (DHEAS) which serve as estrogen precursors for the placenta $(1,2)$. Soon after birth, the outer definitive zone differentiates into $\mathrm{zG}$ and $\mathrm{zF}$, while the inner fetal zone undergoes a marked involution with a corresponding decrease in adrenal androgen levels. Subsequently, at around 6-8 years of age, the emergence of $\mathrm{zR}$ at the corticomedullary junction heralds the onset of adrenarche and the return of adrenal androgen production, including DHEA/DHEAS and androstenedione, leading to axillary and pubic hair development and possibly brain maturation $(3,4)$. While both the $\mathrm{zF}$ and $\mathrm{zR}$ express CYP17, the $\mathrm{zR}$ expresses high levels of the allosteric regulator cytochrome b5 (CYB5) rather than HSD3B2, which favors its 17,20-lyase activity leading to DHEA synthesis. The mechanisms underlying $\mathrm{zR}$ differentiation and the onset of adrenarche 
are still unknown, and genetically tractable animal models that undergo $\mathrm{zR}$ development are nonexistent. For example, the mouse adult adrenal cortex does not express CYP17 and does not develop a functional $\mathrm{zR}(5,6)$. In the region in which the $\mathrm{zR}$ is found in primates, mouse adrenals are characterized by the presence of a transient $\mathrm{X}$-zone, derived from the fetal cortex, which regresses at sexual maturity in males and during the first pregnancy in females $(7,8)$. Lineage-tracing studies have shown that both adult definitive and fetal cortices derive from a common embryonic population of cells expressing $S f 1$, through early activation (E11.5-E12.5) of the specific fetal adrenal enhancer FAdE $(9,10)$. By E14.5, FAdE only drives $S f 1$ expression in cells that will give rise to the fetal X-zone, whereas cells of the definitive cortex use a yet to be identified adult enhancer to maintain $S f 1$ expression.

Once formed, the definitive cortex is continuously replenished with steroidogenic cells to compensate for cell death at the corticomedullary junction and to maintain adrenal size and function. Homeostatic renewal is supported by recruitment of capsular $\mathrm{Rspo}^{+} / \mathrm{Glil}^{+} / \mathrm{SfI}^{-}$stem cells and subcapsular/zG $\mathrm{Shh}^{+} / \mathrm{Wnt}^{+} / \mathrm{SfI}^{+}$progenitor cells in a process that involves RSPO/WNT/ $\beta$-catenin and $\mathrm{SHH}$ signaling pathways. This process eventually induces differentiation of progenitors into $\mathrm{zG}$ cells that are pushed in a centripetal fashion and converted into zF cells (11-14). Stem/progenitor reservoirs are themselves maintained through reciprocal signals, among which Rspo3 induces $W n t 4$ and Shh expression within steroidogenic cells and Shh in turn signals back to recruit capsular cells (14). In the adult cortex, WNT and PKA signaling pathways play essential and complementary roles in functional zonation. Gain- and loss-of-function models have shown that the RSPO/WNT/ $\beta$-catenin pathway is a driver of $z G$ identity, a repressor of $\mathrm{zF}$ identity, and has tumorigenic activity when constitutively activated (14-17). Reciprocally, constitutive activation of cAMP/PKA signaling was shown to inhibit $z G$ fate, trigger $\mathrm{zF}$ identity, and counteract $\beta$-catenin-induced tumorigenesis $(18,19)$. Decreased PKA signaling, resulting from mutations in the $\mathrm{ACTH}$ receptor $\mathrm{MC} 2 \mathrm{R}$, or in its coreceptor MRAP, are associated with cortical atrophy, altered $\mathrm{zF}$ differentiation, and, yet, intact zG (20-22). In contrast, patients with unbuffered PKA activity, resulting from mutations in PRKAR1A (the regulatory subunit of cAMP-dependent PKA), develop micronodular hyperplasia and glucocorticoid excess (ACTH-independent Cushing's syndrome) (23). Strikingly, mice bearing adrenal-specific loss of Prkarla (AdKO model) also develop Cushing's syndrome and show zonation defects consisting of expansion of a third zone, with features typical of both $\mathrm{zF}$ and transient fetal X-zone (19). However, whether these cells originated from the fetal or definitive cortex was unclear.

In the present paper, we evaluated the role of PKA signaling alterations in adrenal homeostasis and differentiation by generating mice with targeted Prkarla genetic ablation specifically in the definitive (adult) cortex or in the transient (fetal) cortex and followed cell dynamics and cortex remodeling by lineage tracing of mutant cells in both sexes. This enabled us to propose that PKA-mediated cortex remodeling not only influences the establishment of adrenarche, but also is part of sexually dimorphic mechanisms that could help to explain the overrepresentation of adrenocortical diseases in women.

\section{Results}

$P K A$ activation in the definitive but not in the fetal cortex induces zonation defects and endocrine hyperactivity. We previously showed that adrenal-specific ablation of Prkarla using the Akr1b7-Cre driver in mice (AdKO mice) resulted in ACTH-independent Cushing's syndrome and centrifugal expansion of large fetal-like cells from the innermost cortex (19). Because the Akr1b7-Cre driver targets recombination to both the definitive (adult) and transient (fetal or X-zone) cortices (24), the origin of these cells has remained elusive. To elucidate the cellular origin of this Cushing's phenotype, we conditionally deleted Prkarla in the (a) fetal cortex, using FAdE-CreERT2 (Cre driven by fetal adrenal enhancer and proximal promoter of $S f 1$ gene) (10), or (b) definitive cortex, using $A S^{\text {Cre }}$ line [Cyp11b2 tm1.1(crebrlt , Cre targeted to the genetic locus of $C y p 11 b 2$, aldosterone synthase] (11) (Figure 1A). These mice will hereafter be referred to as FAdKO (fetal adrenal cortex KO) and DAdKO (definitive adrenal cortex KO). Recombination in the fetal cortex cells was induced at E14.5 by tamoxifen treatment of FAdKO mice, and samples were collected at 3 weeks and 7-14 months. Recombination in the DAdKO model begins at E18.5, at the start of zG differentiation, and continues as zG cells are continuously converted into $\mathrm{zF}$ cells, which migrate to the corticomedullary junction by approximately 1.5 month (excepting X-zone). Samples were collected at 1.5, 3, 7, and 12 months.

Cell fate mapping of cells derived from the fetal or definitive cortex, following deletion of Prkarla, was evaluated by lineage-tracing experiments using FAdE-CreERT2::Prkar1a $a^{f / f t}:: R 26 R^{m T m G / m T m G}$ and $A S^{+/ C r e}:: P r k$ $\operatorname{arl}^{f / f l}:: R 26 R^{m T m G / m T m G}$ trigenic mice, respectively (Figure $1 \mathrm{~A}$ ). Analysis of fetal cortex-derived cells at 3 weeks of age, when the fetal X-zone reached its maximal size, revealed that $\mathrm{GFP}^{+}$cells were restricted to 


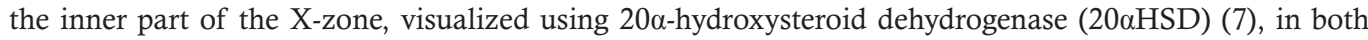
control (Prkar1 $\left.a^{f /+}\right)$ and FAdKO mice (Figure 1B). In contrast, analysis of definitive cortex-derived cells at 1.5 months of age revealed radial columns of $\mathrm{GFP}^{+}$cells extending from the $\mathrm{zG}$ through the $\mathrm{zF}$, with no staining in the X-zone (Figure 1B). At these early stages, Prkarla deletion with either FAdE-CreERT2 or $A S^{+/ C r e}$ had no obvious effect on adrenocortical organization. qPCR analyses showed no major alteration in Prkarla levels in FAdKO adrenals (presumably reflecting the small proportion of fetal cortex-derived cells) but confirmed a robust reduction in Prkarla levels in DAdKO adrenals (Figure 1C). Together, these data establish that fetal and definitive cell lineages never overlap. Furthermore, they show that Prkarla ablation has no obvious morphological effect on adrenal cortices at these early time points.

We next examined the long-term effect of Prkarla deletion in these two models. Surprisingly, analysis of FAdKO adrenals from 7- to 14-month-old mice revealed no morphological alterations of inner cortical cells and no recurrence of fetal-like $20 \alpha \mathrm{HSD}^{+}$cells (Figure 1, D and E, bottom). The lack of histological alterations in this model was also associated with normal basal glucocorticoid plasma levels and a normal response to dexamethasone-mediated hypothalamic-pituitary-adrenal blockade (Figure 1F). In contrast, analysis of DAdKO adrenals showed alterations reminiscent of AdKO mice (19). Indeed, DAdKO adrenals showed a time-dependent decrease in the size of the $\mathrm{zF}$ and a concomitant expansion of the hypertrophic/ eosinophilic $20 \mathrm{aHSD}^{+}$cells in the innermost cortex (Figure 1, D and E, top). In addition to the dramatic morphological changes, DAdKO mice also demonstrated overt pituitary-independent Cushing's syndrome. At 3 and 7 months of age, DAdKO mice showed baseline hypercorticosteronemia and complete resistance to dexamethasone-induced hypothalamic-pituitary-adrenal axis suppression, associated to decreased circulating ACTH concentrations (Figure 1F). Taken together, these data suggest that the Cushing's syndrome that arises following loss of Prkarla results from cells derived from the definitive cortex.

Constitutive PKA activation induces conversion of inner $z F$ into functional $z R$. While we previously showed an expansion of a fetal-like zone in AdKO mice (19), those studies could not establish the cellular origin of this new zone. To address this question, we combined lineage-tracing analysis with immunohistochemistry for $20 \alpha \mathrm{HSD}$ in 3- to 7-month-old mice. As expected, in control mice, cells comprising the X-zone $\left(20 \alpha \mathrm{HSD}^{+}, \mathrm{GFP}^{-}\right)$were completely distinct from cells of the definitive cortex $\left(20 \alpha \mathrm{HSD}^{-}, \mathrm{GFP}^{+}\right)$. In contrast, in DAdKO mice, the fetal-like zone was composed of cells that expressed both $20 \alpha \mathrm{HSD}$ and GFP (Figure 2A). This result demonstrates that this fetal-like zone arose from the conversion of $\mathrm{zF}$ cells and is distinct from the X-zone. We next carried out qPCR analyses to measure time-dependent expression of 2 X-zone markers (Akr1c18, encoding $20 \alpha \mathrm{HSD}$, and $P i k 3 c 2 g$, ref. 25) during expansion of the fetal-like zone in DAdKO females and spontaneous regression of the X-zone in controls (which occurs at $\sim 5$ months in virgin females, ref. 7). Molecular analysis revealed a marked increase in Akr1c18 expression in DAd$\mathrm{KO}$ adrenals from 7-month-old females (Figure 2B). In contrast, analysis of Pik3c2g expression revealed a marked decrease in expression at 3 months in DAdKO, with undetectable expression in both control and DAdKO adrenals at 7 months, as expected following X-zone regression in controls (Figure 2B). This observation supports the conclusion that expansion of the $20 \alpha \mathrm{HSD}^{+}$fetal-like zone in DAdKO adrenals is independent of the X-zone and is paralleled by a premature regression of the X-zone.

We next sought to gain additional insight into the steroidogenic potential of this fetal-like zone. Immunostaining of adrenal sections from 7-month-old WT and DAdKO females showed that this zone expressed very low levels of 3 $\beta$-hydroxysteroid dehydrogenases (HSD3Bs) and high levels of CYB5 and CYP17, which is normally absent from the adult mouse adrenal gland (Figure $2 \mathrm{C}$ ). The results were confirmed using Western blot and qPCR analysis (Figure 2, D and E). qPCR analysis also revealed increased expression of Sult1e1, which can be involved in DHEA sulfation (26) (Figure 2D). Taken together, decreased expression of HSD3B (HSD3B2 in humans, HSD3B1 in mice), expression of CYP17 and CYB5 (its allosteric regulator), and expression of the potential for steroid sulfation are reminiscent of the human $\mathrm{zR}(5,27-32)$. To investigate the functional capacity of these putative zR-like cells, we analyzed concentrations of steroids relying on CYP17 activity (Figure 2F). Remarkably, we observed a robust increase in cortisol, 21-deoxycortisol, androstenedione, DHEA, and DHEAS concentrations in plasma and/or adrenals from DAdKO mice (Figure 2F). Altogether, these data show that mice with constitutive PKA activation in cells derived from the definitive cortex develop a new innermost zone, which exhibits features that are highly reminiscent of the human $\mathrm{zF}$ and $\mathrm{zR}$.

Mechanisms underlying endocrine hyperactivity in DAdKO mice. Activation of steroidogenesis by ACTH/ PKA is considered to be mainly dependent on transcriptional stimulation of steroidogenic genes. However, steroidogenic output also depends on the availability of cholesterol, which is provided by cellular uptake of 
A
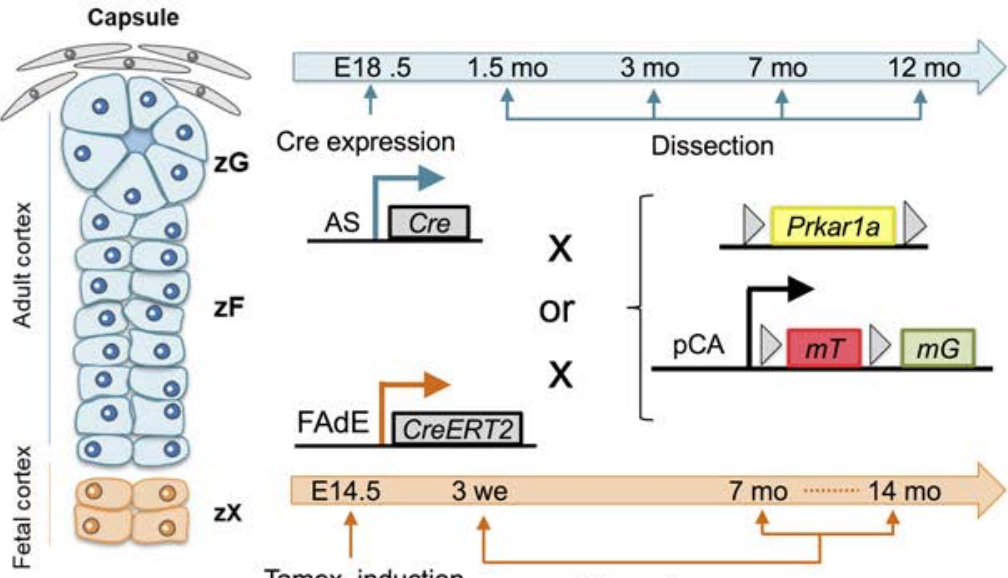

C

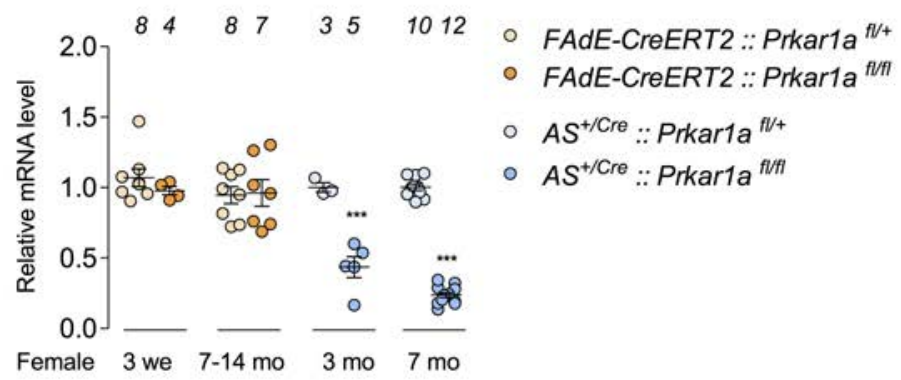

E

E $\quad W T$

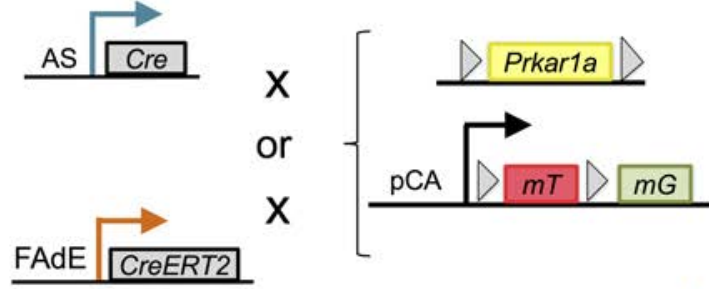

$x$

Tamox. induction

Dissection

\section{Prkar1a}

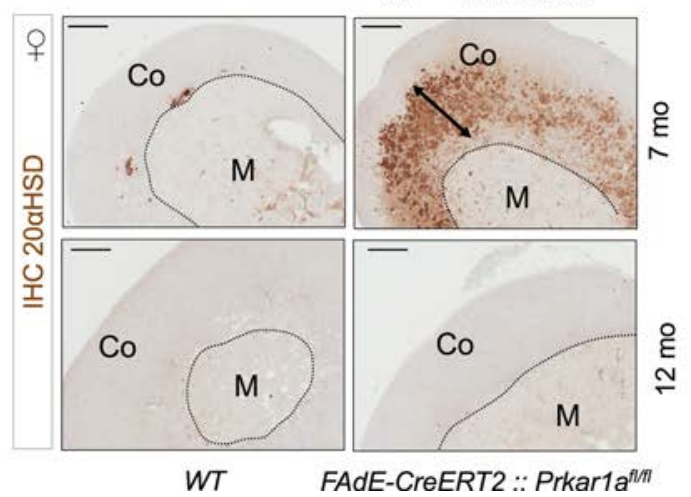

B

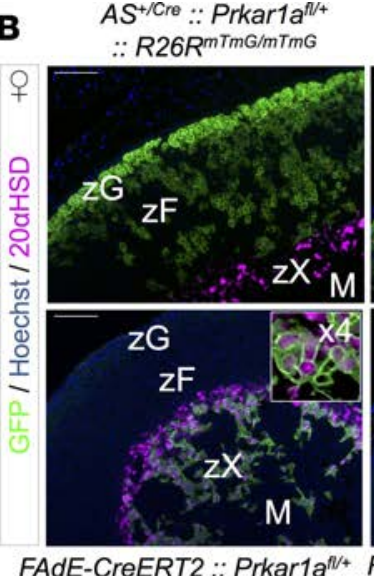

$A S^{+/ C r e}::$ Prkar1a ${ }^{n / 4}$

$\because R 26 R^{m T m G / m T m G}$

FAdE-CreERT2 :: Prkar1

D

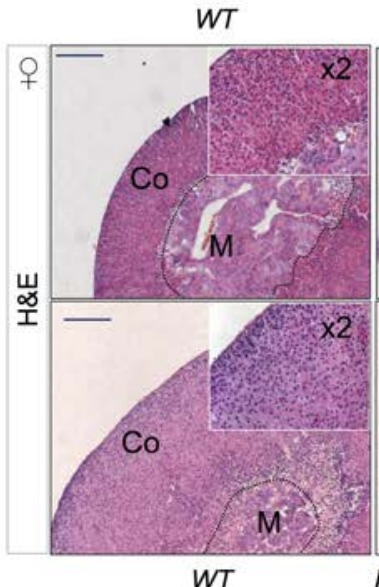

$\mathbf{F}$
$A S^{+/ C r e}::$ Prkar1a $a^{\text {Int }}$

: R26RmTmG/mTmG
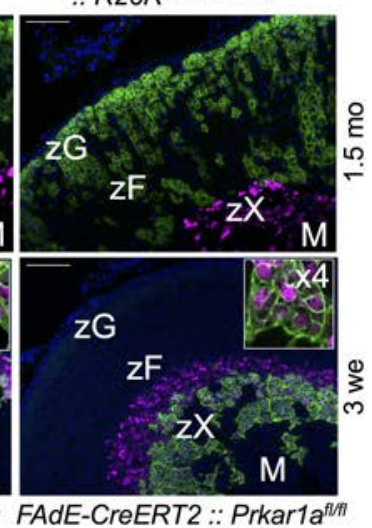

:: R26R $R^{m T m G / m T m G}$

$A S^{+/ C r e}::$ Prkar1a ${ }^{\text {f/m }}$

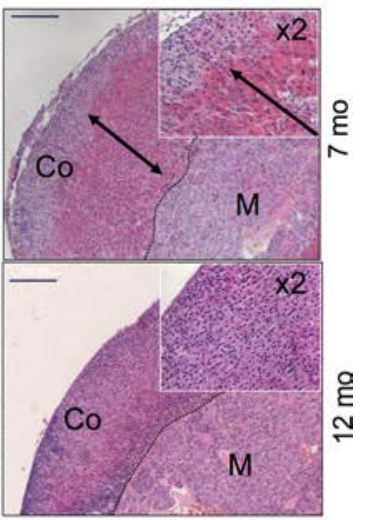

FAdE-CreERT2 :: Prkar1a $a^{f / A}$

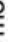

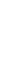

\section{.}


lipoproteins, mobilization of intracellular stores, and de novo synthesis (33). To test which biosynthetic step(s) may account for endocrine hypercorticosteronemia in DAdKO mice, we carried out qPCR and genomewide expression analyses. Strikingly, although Star and Cyp21a1 mRNAs levels were upregulated 2-fold and CYP11A1 and CYP21 proteins were slightly increased in immunohistochemistry (Figure 3, A and B), most steroidogenic genes were either unaltered or modestly repressed in DAdKO adrenals (Figure 3A). This suggested that increased corticosterone production is not the result of increased expression of key steroidogenic enzymes. In contrast, analysis of genes important for supplying adrenal cells with lipoprotein-derived cholesterol substrate ( $L d l r$ and Scarb1) and detoxifying steroidogenesis byproducts (Akr1b7) (34) revealed a more dramatic 5- to 6-fold upregulation (Figure 3A). Moreover, microarray analysis revealed that nearly every gene involved in de novo cholesterol synthesis was upregulated in DAdKO adrenals compared with control adrenals (Figure 3C). Gene set enrichment analyses (GSEA) analysis of a "cholesterol synthesis" gene set showed a robust positive enrichment in DAdKO adrenals (Figure 3D), which was confirmed by strong upregulation of mRNA levels for genes (3- to 8-fold) encoding key enzymes of the mevalonate pathway and for their common transcriptional regulator Srebf2 in qPCR (Figure 3E). Thus, we concluded that increased glucocorticoid production in mice with adrenal-specific constitutive PKA activation could be largely the result of enhanced cholesterol uptake and de novo synthesis, allowing for increased substrate for downstream steroidogenic enzymes. Interestingly, even though we confirmed that $\mathrm{zG}$ differentiation was markedly impaired by chronic PKA activation (Supplemental Figure 1; supplemental material available online with this article; https://doi.org/10.1172/jci. insight.98394DS1), there was a significant increase in plasma aldosterone concentration in DAdKO mice com-

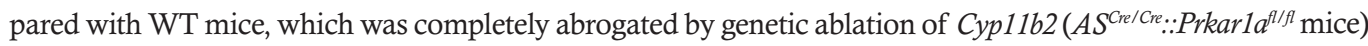
(Figure 3F). Importantly, plasma renin activity was decreased in DAdKO mice, indicating primary hyperaldosteronism, while, as expected, lack of CYP11B2 resulted in a strong rise of plasma renin activity (Figure 3F). Note that the impairment of Cyp11b2 expression was milder in DAdKO adrenals than in Sf1-Cre::Prkar1a ${ }^{\text {fl/fl }}$ adrenals (50\% vs. $95 \%$, ref. 18) (Supplemental Figure 1D), maintaining aldosterone synthase activity above the critical threshold. Altogether, these data suggest that increased cholesterol supply, leading to increased flux through the steroidogenic pathways, is sufficient to induce overproduction of both corticosterone and aldosterone in response to chronic PKA activation in cells of the definitive cortex. Interestingly, when compared with angiotensin II and plasma potassium, ACTH acting through PKA signaling was long regarded as a minor hormonal regulator of aldosterone secretion. However, there is growing evidence that ultrasensitivity to ACTH may account for primary hyperaldosteronism as a major cause of secondary hypertension $(35,36)$. Primary hyperaldosteronism found in DAdKO mice thus provides the first demonstration to our knowledge that genetic alteration in PKA holoenzyme subunits may affect aldosterone production.

Cushing's syndrome and comorbidities in DAdKO mice. Glucocorticoid excess in Cushing's syndrome leads to severe comorbidity, primarily affecting glucose, fat, and protein metabolism. This results in insulin resistance, diabetes, obesity, skin fragility, and muscle atrophy $(37,38)$. Compared with control mice, 7-monthold DAdKO females exhibited both obesity and skin alterations leading to alopecia (Figure 4A). DAdKO mice started to gain weight faster than controls, between 3 and 3.5 months of age, and this difference was exacerbated with time, with an almost doubling of body weight at 7 months (Figure 4B). Analysis of body composition using EchoMRI showed that weight gain in mutants resulted from a 4 -fold increase in the fat mass (Figure 4C). Female DAdKO mice also suffered from muscle wasting, illustrated by a 20\% drop in gastrocnemius weight (Figure 4C). They also showed higher fasting glycemia, most likely resulting from a combination of increased gluconeogenesis and insulin resistance (Figure 4D).

Onset of Cushing's syndrome and cortex alterations are delayed by androgens. In stark contrast to DAdKO females, which demonstrated overt Cushing's syndrome by 3 months of age, DAdKO males developed phenotypic alterations between 7 and 12 months (Figure 4, E and F, and Supplemental Figure 2). Lineage-tracing studies indicated that complete GFP staining of the adult cortex took up to 7 months in mutant males (Figure 4E), while the cortex was fully GFP labeled as early as 3 months in females (Figure 2A). These findings indicated that adrenocortical turnover is slower in male mice compared with female mice, which may partially explain the delayed in manifesting the phenotypic changes. Consistent with this observation, qPCR analysis showed that Prkarla deletion was similarly delayed in male adrenals (Figure 4G). Accordingly, deletion of Prkarla in adult adrenocortical lineage resulted in $\mathrm{zF}$ disorganization and expansion of $20 \alpha \mathrm{HSD}^{+} / \mathrm{GFP}^{+}$ inner zone after 3 months in females, while it took 12 months in males (Figure 4E). Independent of the slower adrenocortical turnover in male mice, the marked delay in the onset of Cushingoid features suggested that male sex steroids might play a role in suppressing the emergence of this phenotype. 


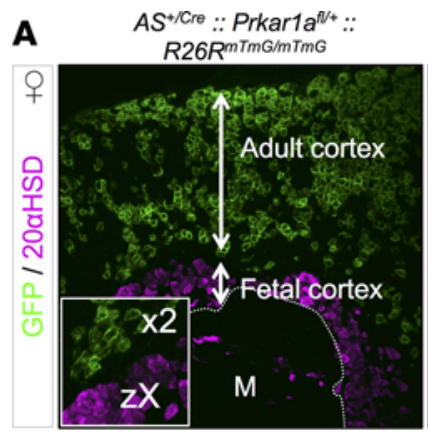

C
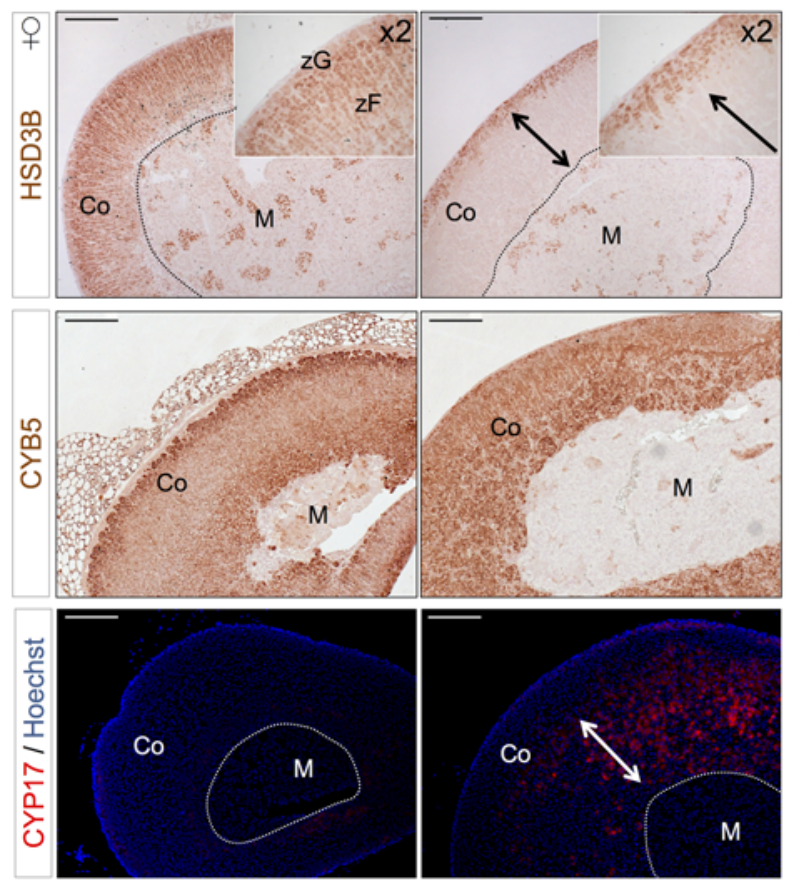

$\mathbf{F}$

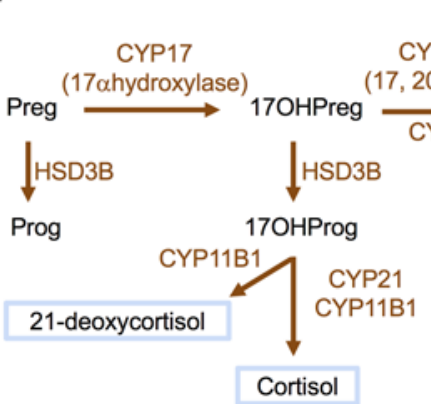

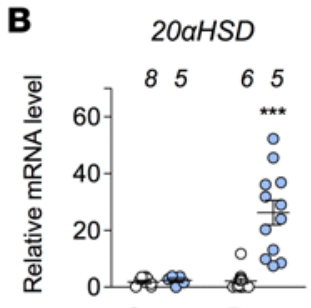

Female $3 \mathrm{mo} \quad 7 \mathrm{mo}$

D

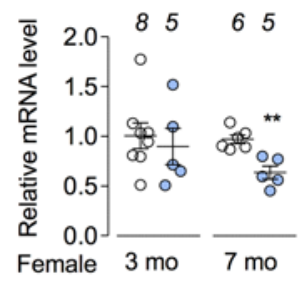

Cyb5a

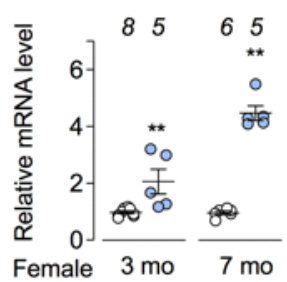

Pik3c2g

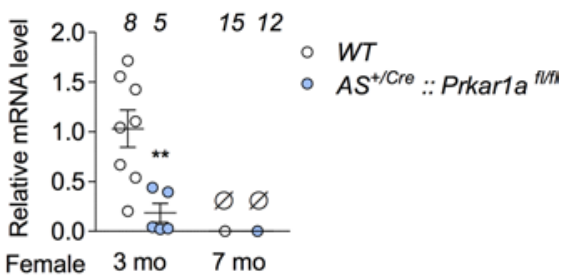

\section{Cyp17a1}

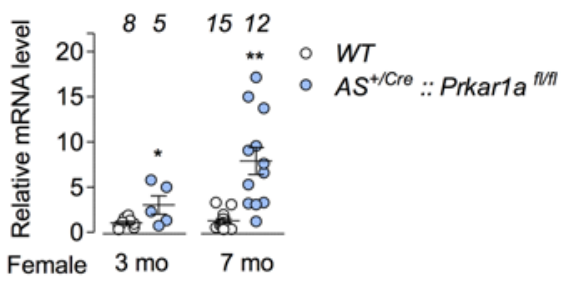

Sult1e1

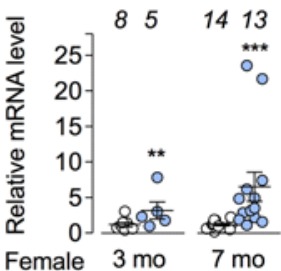

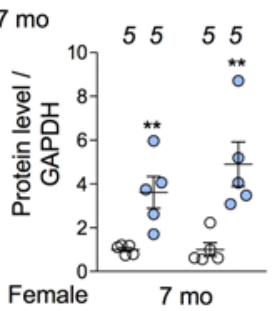

- WT $\circ A S^{+/ C r e}::$ Prkar1a ${ }^{f / n}$

Figure 2. Inner hyperplasia formed in $\mathbf{A S}^{+/ / \text {re }}:$ Prkar1afl/f mice (DAdKO) has reticularis characteristics. (A) Confocal analysis of coimmunofluorescent

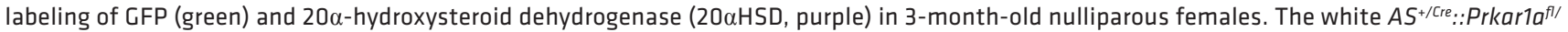

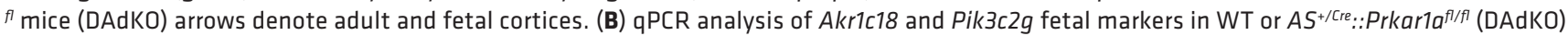
female adrenals. (C) Immunostaining and immunofluorescent analyses of HSD3B, CYB5A, and CYP17 from DAdKO 7-month-old females. The black and white double arrows denote inner hyperplasia. The black and white dots represent the border between the cortex and the medulla. (D) qPCR analysis confirms downregulation of Hsd3b1, upregulation of Cyp17a1 and Cyb5a, and, moreover, shows increased expression of Sult1e1 in DAdKO adrenals. (E) Western blot analysis of CYP17 and CYB5A protein accumulation in adrenal extracts from WT and mutant mice. Graphs showed quantification normalized to quantification of GAPDH signal. (F) Cortisol, 21-deoxycortisol, and $\Delta 4$ androstenedione levels were measured by mass spectrometry in blood, dehydroepiandrosterone (DHEA) levels were measured by ELISA in adrenal extracts from adult mice ( $>7$ months), and dehydroepiandrosterone sulfate (DHEAS) levels were measured by RIA in blood. zG, zona glomerulosa; zF, zona fasciculata; zX, X-zone; Co, cortex; M, medulla. Lines in dot plots represent the mean \pm SEM. $\emptyset$, under detection threshold. Statistical analyses were conducted by Student's $t$ test. ${ }^{*} P<0.05,{ }^{*} P<$ $0.01,{ }^{* *} P<0.001$. The number of samples is indicated above dot plots. Scale bars: $200 \mu \mathrm{m}$. Original magnification: $\times 2$ (insets, A); $\times 4$ (insets, C). 
A Steroidogenesis

- $W T$

- $A S^{+/ C r e}::$ Prkar1a flft

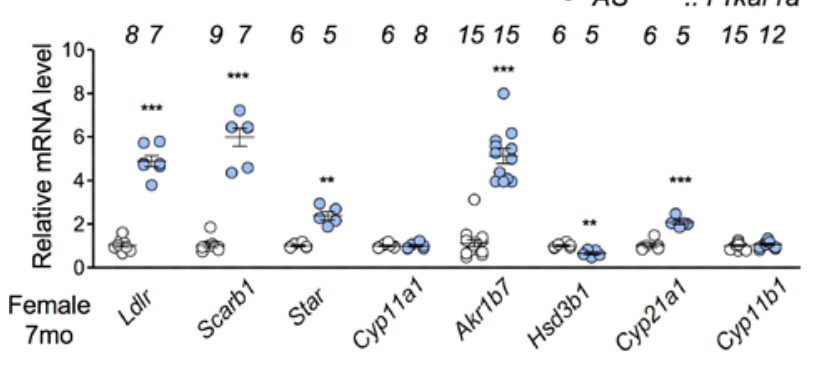

C

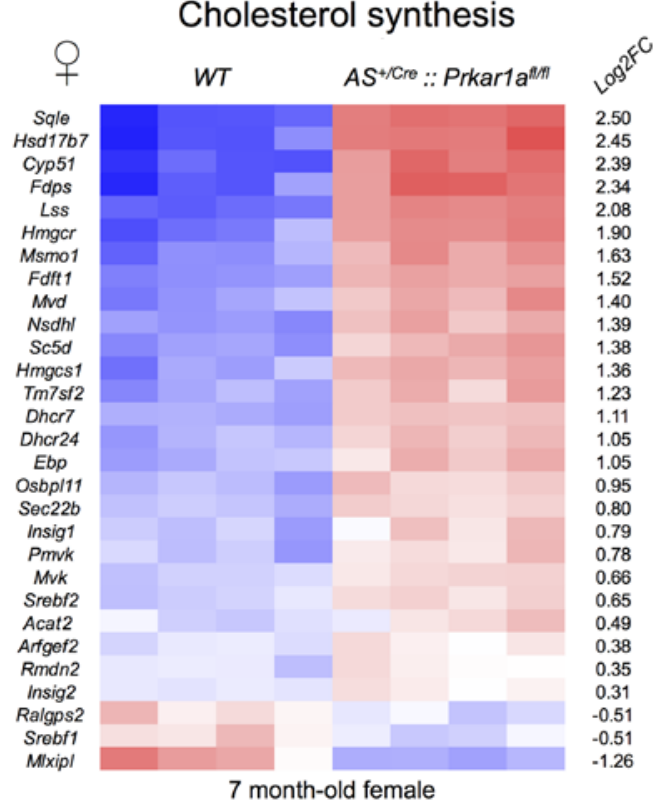

E

Cholesterol synthesis

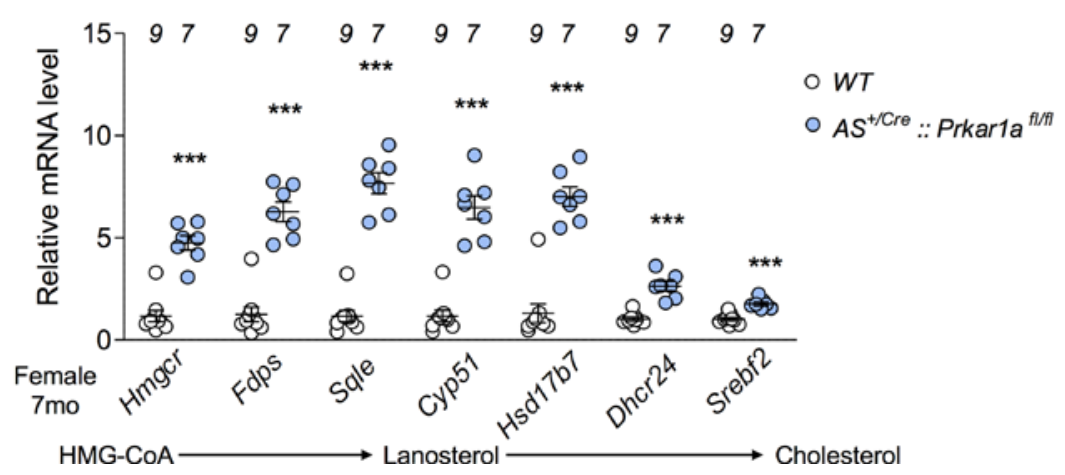

B

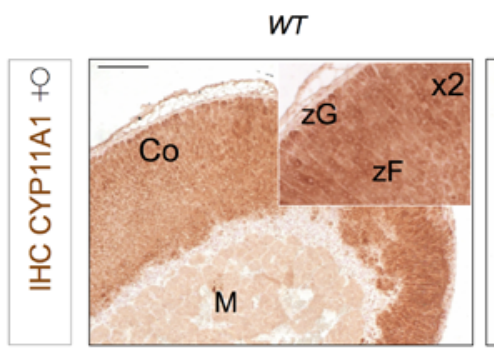
$A S^{+/ C r e}::$ Prkar1a $a^{A m} \quad 7 \mathrm{ma}$
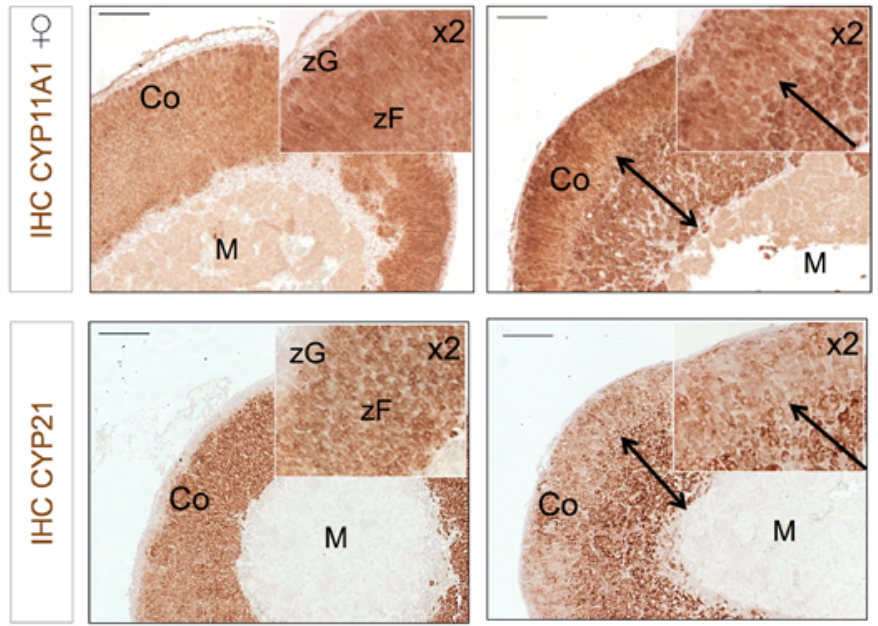

D

Enrichment plot :

Cholesterol Synthesis

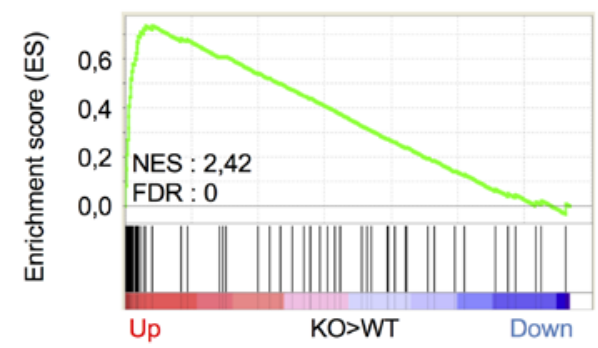

$\mathbf{F}$

Aldosterone

Renin activity
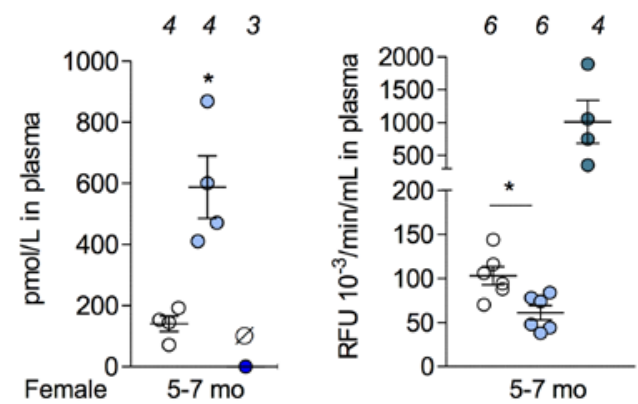

○ WT

- $A S^{C r e / C r e}::$ Prkar1a ${ }^{\text {flff }}$

- $A S^{+/ C r e}::$ Prkar1a ${ }^{\text {flfl }}$ - $A S^{C r e / C r e}$

Figure 3. Endocrine overactivity is associated with upregulation of steroidogenesis pathway and cholesterol synthesis. (A) qPCR analysis of steroidogenic gene expression in adrenals of DAdKO 7-month-old females. eChol/iChol, extra/intracellular cholesterol; Preg., pregnenolone; Prog., progesterone; DOC, deoxycorticosterone; Cort., corticosterone. (B) Immunostaining of CYP11A1 and CYP21 in adrenal sections from DAdKO 7-month-old females. (C) Color-coded heatmap shows expression of the 29 genes that are significantly deregulated (adjusted $P=0.05$ ) relative to the cholesterol synthesis pathway. (D) Gene set enrichment analyses were performed using signatures gene set of cholesterol synthesis pathway by comparing gene expression data from WT and DAdKO female adrenals. Normalized enrichment score (NES) and false discovery rates (FDR) are shown on plots. The enrichment score plot corresponds to the 59 genes listed in Supplemental Table 3. (E) qPCR analysis of genes involved in de novo cholesterol synthesis in adrenals from

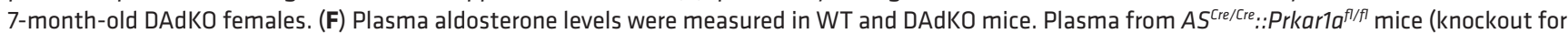
Prkar1a and Cyp11b2 [aldosterone synthase]) was used as negative control. Plasma renin activity rate was compared in WT and DAdKO mice. ASCre/cre mice were used as positive control for renin-angiotensin system upregulation. Lines in dot plots represent the mean $\pm \mathrm{SEM}$. $\mathrm{zG}$, zona glomerulosa; $\mathrm{zF}$, zona fasciculata; $\mathrm{ZX}$, X-zone; Co, cortex; $\mathrm{M}$, medulla. The black double arrows focus on hyperplasia. If not otherwise mentioned, all statistical analyses were conducted by Student's $t$ test. ${ }^{*} P<0.05,{ }^{* *} P<0.01,{ }^{* *} P<0.001$. The number of samples is indicated above dot plots. Scale bars: $200 \mu \mathrm{m}$. Original magnification: $\times 2$ (insets, B). 
To test this hypothesis, DAdKO and control male mice were castrated at 5 months, and their adrenal phenotypes were evaluated 2 months later (Figure 5A). As expected, in control males, castration at 2 months was sufficient to induce recurrence of a secondary X-zone $\left(20 \alpha \mathrm{HSD}^{+}\right)$, which was absent from sham animals (Figure 5B, left). Remarkably, these $20 \alpha \mathrm{HSD}^{+}$cells were clearly distinct from cells derived from the definitive cortex, marked by GFP. In contrast, castration of DAdKO males induced formation of a large $20 \alpha \mathrm{HSD}^{+} /$ $\mathrm{GFP}^{+}$zone, which was absent from sham mutants (Figure $5 \mathrm{~B}$, right). This phenotype, reminiscent of the 7-month-old female DAdKO adrenal, could be almost completely reversed by dihydrotestosterone (DHT) supplementation for 6 weeks, indicating that androgens were directly responsible for the emergence of this zone and possibly for the delayed acquisition of the phenotype in male mice (Figure 5). Consistent with these findings, qPCR showed that Akr1c18 was upregulated following both gonadectomy-induced (GDX-induced) X-zone formation (in control mice) or GDX-induced zR-like zone formation (in DAdKO mice), while elevated expression of $P i k 3 c 2 g$ was only seen following GDX-induced X-zone formation (in control mice) (Figure 5C). To evaluate to what degree the castration-induced phenotype in male DAdKO mice resembled the female DAdKO phenotype, we explored basal and dexamethasone-suppressed corticosterone levels in sham and GDX $( \pm \mathrm{DHT})$ males. As expected, we found that basal and dexamethasone-suppressed corticosterone levels were unaffected in 7-month-old DAdKO sham males compared with controls (Figure 5D). In a similar fashion, GDX did not alter corticosterone levels in control males. In contrast, GDX in aged-matched DAdKO males triggered development of an overt ACTH-independent Cushing's syndrome (basal hypercorticosteronemia and resistance to dexamethasone suppression test) that was fully reversed by androgen replacement (Figure 5D, GDX, GDX ${ }^{+} \mathrm{DHT}$ ). We further conducted a comparative microarray analysis of whole-genome expression in adrenals of 7-month-old mice from the 6 groups (DAdKO female, WT female, DAdKO male, WT male, GDX DAdKO male, and GDX WT male). Consistent with the GDX-unveiled KO phenotype in males, 256 genes were found upregulated and 183 were found downregulated in response to Prkarla deletion in GDX males, while very few (only 10 and 2, respectively) were differentially expressed in intact KO males (Supplemental Figure 3A). Principal component analysis and unsupervised gene clustering showed that experimental groups segregated into 3 clusters: a Cushing cluster gathering DAdKO female and GDX DAdKO male subclusters, a female cluster containing WT female and GDX WT male samples, and a male cluster containing WT and DAdKO male samples (Supplemental Figure 3B). As shown for hypercorticism in DAdKO females (Figure 3A), Cushing's syndrome unveiled by castration in DAdKO males was correlated with upregulation of lipoprotein receptors and steroidogenic genes. As expected, this effect was reversed by androgen supplementation (Supplemental Figure 3C). In contrast to females, however, endocrine hyperactivity in castrated DAdKO males seemed less dependent on upregulation of de novo cholesterol synthesis genes. Although contribution of the mevalonate pathway remains to be functionally assessed, this was reminiscent of recent transcriptomic analyses in rat adrenals, suggesting that genes related to cholesterol homeostasis were more sensitive to hormonal regulation in females than in males (39). Altogether, these data establish that Prkarla genetic ablation in the adult adrenocortical lineage leads to endocrine hyperactivity and conversion of inner $\mathrm{zF}$ into a reticularis-like zone, which is unrelated to the X-zone. This phenomenon relies partly on sex-dependent mechanisms of cortical renewal, on which the male androgenic milieu exerts a repressive action.

PKA-dependent accelerated cortex renewal is counteracted by androgens. To investigate the mechanism(s) that support cell renewal upon constitutive PKA activation, we analyzed the rate of recombination in lineage-tracing experiments. Lineage tracing at 3 months showed that, in control females, numerous radial $\mathrm{GFP}^{+}$columns of $\mathrm{zF}$ cells did not reach the medulla and even some cortical areas were still nonrecombined $\left(\right.$ Tomato $^{+}$). In contrast, Prkarla genetic ablation resulted in complete GFP labeling of the cortices in agematched DAdKO glands (Figure 6A). Quantification of GFP mRNA levels confirmed that constitutive activation of PKA let to a 3-fold increase in the rate of recombination in female mice (Figure 6B). To test whether mutant adrenals showed changes in cell proliferation, we performed a BrdU pulse-chase experiment and also quantified Pcna transcripts in 7-month-old females. Surprisingly, whereas the number of BrdU-labeled cells was not altered, Pcna expression was even decreased in DAdKO adrenals (Supplemental Figure 4A). These data indicated that accelerated lineage tracing (marking cell renewal) after Prkarla ablation was not accompanied by an increase in cell division but rather an acceleration of the centripetal differentiation process. The adrenal capsule and subcapsular/zG region harbor stem/progenitor cells, the progeny of which contribute to the homeostatic maintenance and renewal of the cortex (40). We used our microarray data to evaluate the role of constitutive PKA activation on progenitor compartment homeosta- 
A

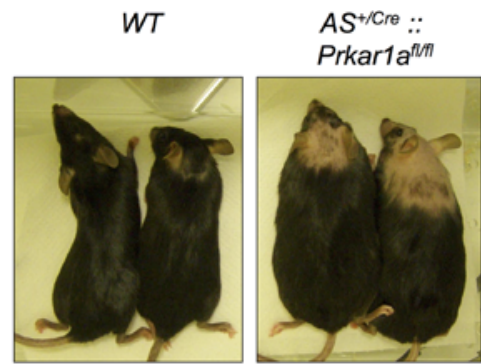

B

- $W T \quad \circ A S^{+/ \text {Cre }:: P r k a r 1 a}{ }^{\text {flft }}$

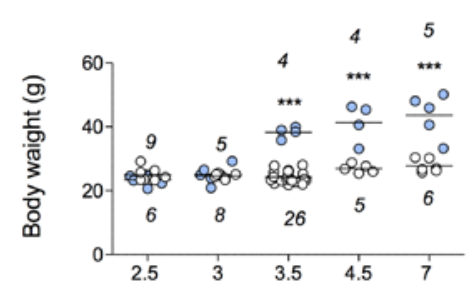

Female $\quad$ Age (months)

\section{Lean mass}

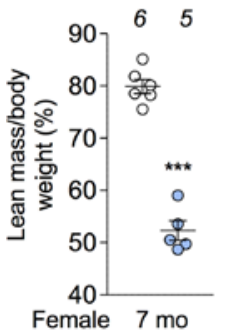

Fat mass

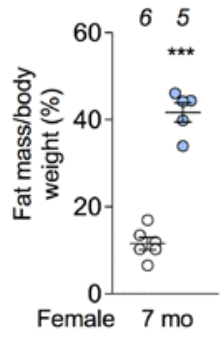

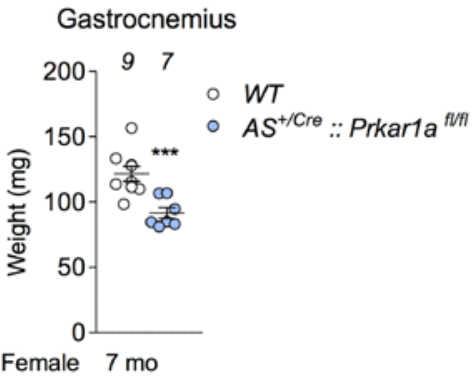

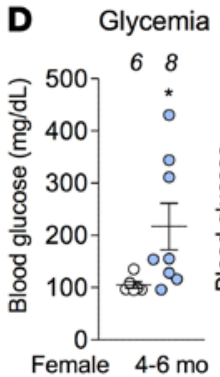

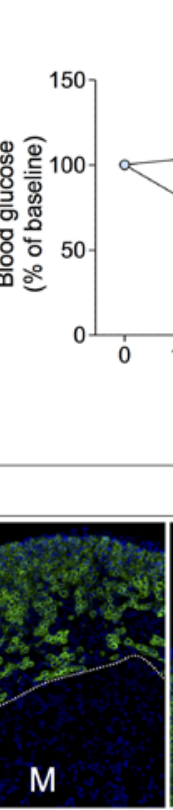

$7 \mathrm{mo}$

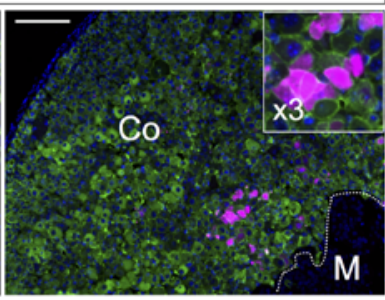

$12 \mathrm{mo}$
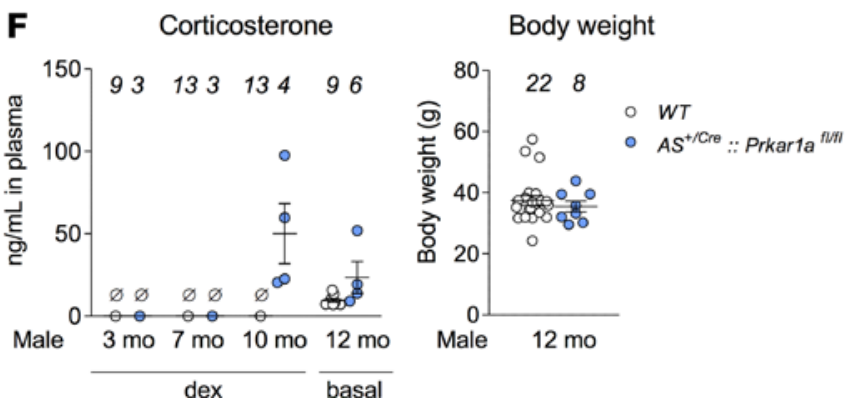

G

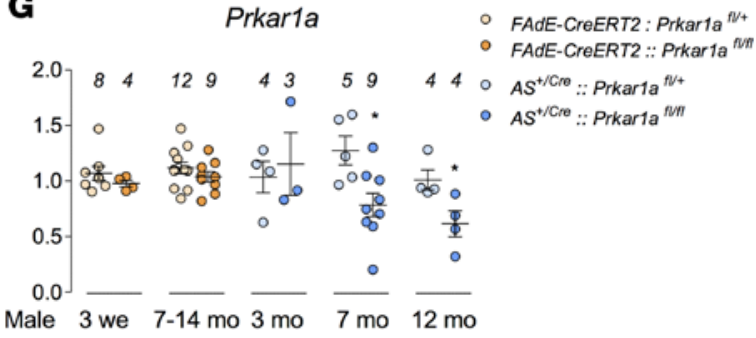

Figure 4. Cushing's syndrome due to loss of R1 $\alpha$ in the adult cortex develops in a sex-dependent manner. (A-D) Female data. (A) Image of 7-month-old DAdKO females showing fat distribution compared with WT. (B) Body weight (g) follow-up of WT and DAdKO females (mean \pm SEM) from 2.5-7 months of age. (C) EchoMRI analysis of lean and fat mass distribution (\%) in 7-month-old females and changes (mg) in gastrocnemius weight (mean \pm SEM). (D) Fasted blood glucose concentration $(\mathrm{mg} / \mathrm{dl})$ and insulin tolerance test (ITT, \% of baseline) in DAdKO females (mean \pm SEM); statistical analysis was conducted by 2-way ANOVA followed by Bonferroni's test. (E-G) Male data. (E) Coimmunofluorescent labeling of GFP

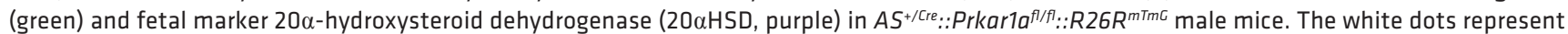
the border between the cortex and the medulla. ZX, X-zone; Co, cortex; M, medulla. (F) Plasma corticosterone levels in basal conditions and after dexamethasone suppression test (dex) and body weight of DAdKO males. $\emptyset$, under detection threshold. (G) qPCR follow-up of Prkar7a expression in adrenals from FAdKO and DAdKO males. Lines in dot plots represent the mean relative quantification of Prkar1a expression (relative to the control counterparts, white bars) \pm SEM. If not otherwise mentioned, all statistical analyses were conducted by Student's $t$ test. ${ }^{*} P<0.05,{ }^{* * *} P<0.001$. The number of samples is indicated in curves or above dot plots. Scale bars: $200 \mu \mathrm{m}$. 
A

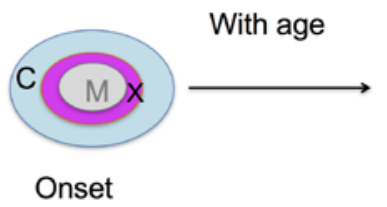

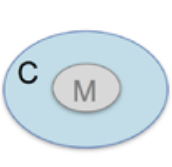

Regression

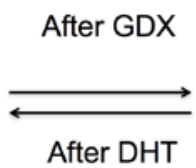

After DHT
Recurrence

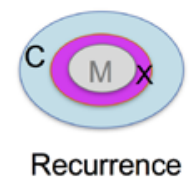

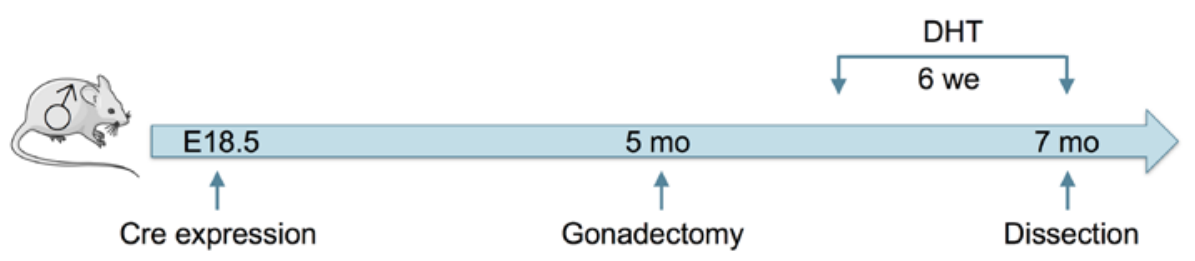
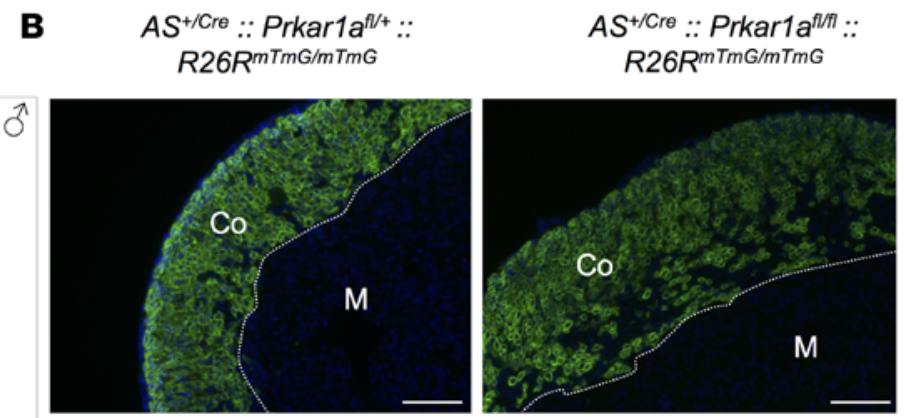

C $\operatorname{Akr1c18}(20 \alpha H S D)$

Pik3c2g

$\begin{array}{llll}85 & 47 & 46\end{array}$

$\begin{array}{llll}85 & 47 & 46\end{array}$

$7 \mathrm{mo}$
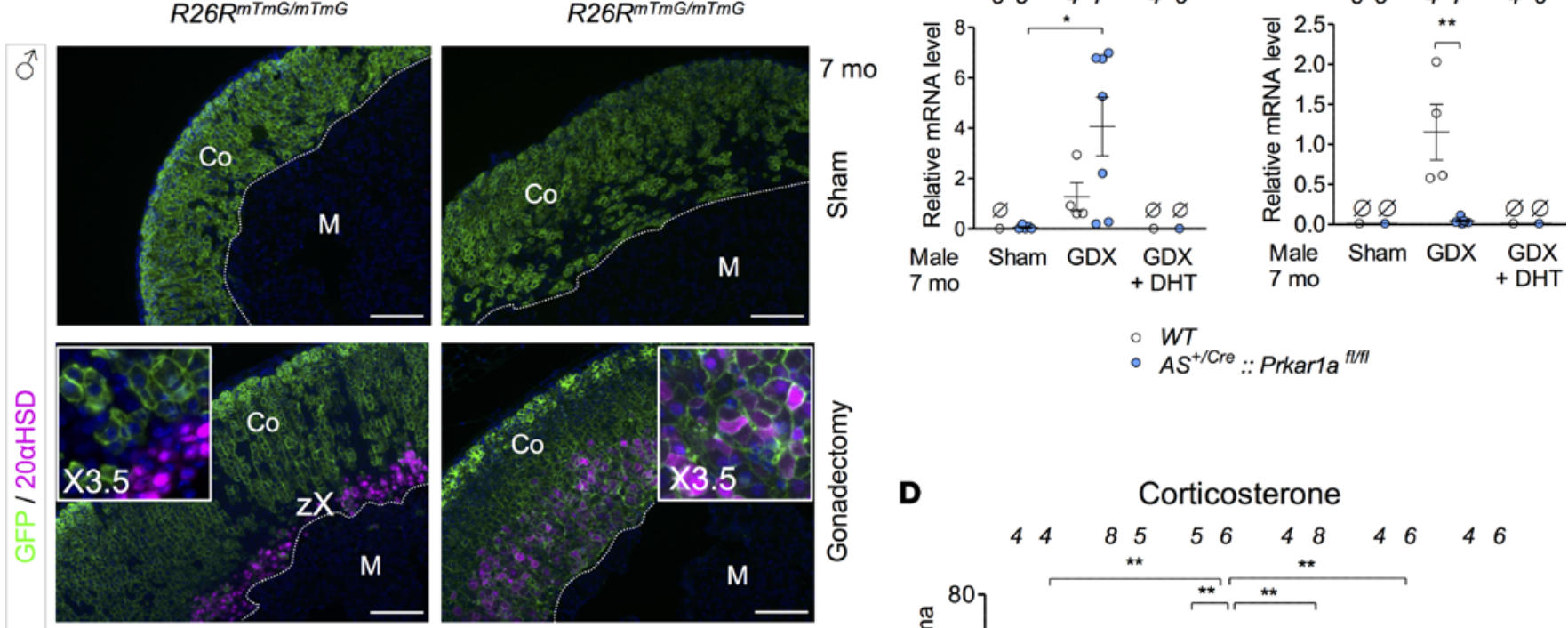

D

- $W T$

- $A S^{+/ C r e}::$ Prkar1a ${ }^{f / f l}$
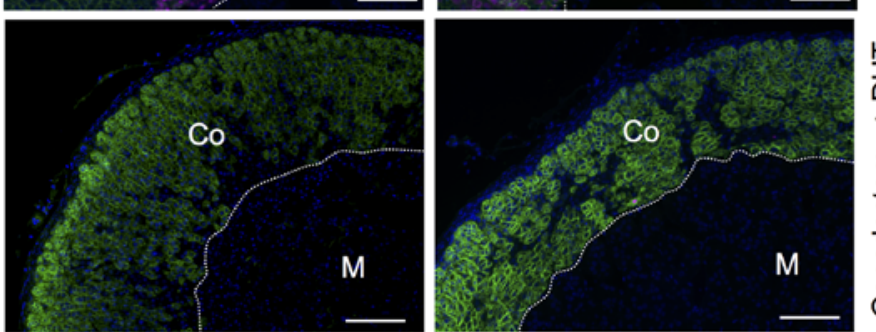

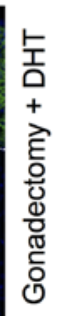

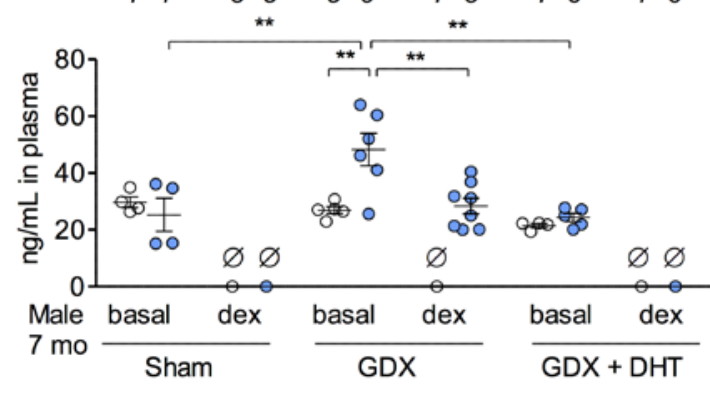

-WT

- $A S^{+/ C r e}::$ Prkar1a flft

Figure 5. Reticularis-like formation may be stimulated by castration in mutant males. (A) Experimental set up. In male mice, $X$-zone regresses at puberty and secondary X-zone regenerates after gonadectomy (CDX) or regresses upon androgenic (dihydrotestosterone [DHT]) supplementation (GDX+DHT). Prkar1a was deleted in adult cortex (from E18.5), and castration was performed at 5 months, i.e., 2 months before dissection, at 7 months. DHT treatment was performed during the last 6 weeks. (B) Effects of gonadectomy and androgen replacement on X-zone and reticularis-like occurrence. Coimmunofluorescent labeling of GFP and $20 \alpha$-hydroxysteroid dehydrogenase (20 $\alpha \mathrm{HSD})$ in adrenal from controls (AS+//re::Pr-

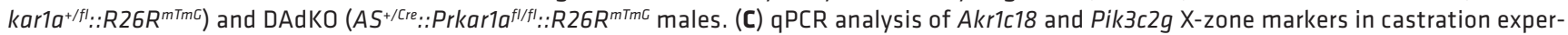
iments. Lines in dot plots represent the mean relative quantification of gene expression (relative to the gonadectomized WT) \pm SEM. (D) Plasma corticosterone levels in basal conditions or after dexamethasone suppression test (dex) in gonadectomized (GDX) DAdKO males in comparison to WT. Lines in dot plots represent the mean \pm SEM. Statistical analyses were conducted by 1 -way ANOVA followed by Bonferroni's test. ${ }^{*} P<0.05$, ${ }^{*} P<0.01$. $\emptyset$, under detection threshold. $\mathrm{zX}, \mathrm{X}$-zone; Co, cortex; M, medulla. The white dots represent the border between the cortex and the medulla. The number of samples is indicated above dot plots. Scale bars: $200 \mu \mathrm{m}$. Original magnification: $\times 3.5$ (insets, B). 
sis. We extracted expression levels of a list of 28 genes expressed or functionally linked to the progenitor compartment and found that 18 of them were significantly ( $t$ test, adjusted $P<0.05$ ) downregulated in Prkarla-knockout adrenals compared with WT adrenals (Supplemental Figure 4B). We further confirmed by qPCR that Prkarla ablation in steroidogenic cells resulted in the downregulation of all stem/progenitor markers $(12,14,41-43)$ from either capsular or subcapsular compartments (Supplemental Figure 4C). The $\mathrm{SHH}$ and RSPO/WNT/ $\beta$-catenin signaling pathways play an essential role in adult cortex maintenance and renewal $(12,14)$. Interestingly, even though the mutant cortex was fully recombined by 3 months, expression of Shh, Gli1, Wnt4, and Rspo3 was unaltered at this stage in DAdKO female adrenals; however, we observed a 50\% decrease in their expression at 7 months (Supplemental Figure 4D). This suggests that PKA activation does not directly inhibit expression of regulators of progenitor homeostasis, but rather accelerates cortical renewal, eventually resulting in relative depletion of the progenitors pool.

Delayed acquisition of phenotypic features in male DAdKO mice suggested that androgens may be inhibiting cortical cell turnover. Consistent with this, recombination evaluated by GFP accumulation was significantly increased following GDX and decreased after DHT supplementation (Figure 6C). This was also associated with decreased expression of Shh and Rspo3, as in female mutants, which was restored by DHT supplementation (Figure 6D). Recently, WNT/ $\beta$-catenin signaling has been shown to inhibit PKA signaling and $\mathrm{zF}$ differentiation, promoting the undifferentiated state of progenitors (17). Interestingly, the androgen receptor has been shown to signal through and cooperate with WNT/ $\beta$-catenin signaling in prostate cancer $(44,45)$. We thus hypothesized that the inhibitory effect of androgens on cortical cell turnover could rely on stimulation of WNT signaling, which would repress PKA activation. Consistent with this hypothesis, expression of $\beta$-catenin target genes Axin2, Lef1, and Lrp5 decreased after GDX and was restored after DHT supplementation (Figure 6E). Although the mechanism underlying the positive effect of androgens on WNT signaling is still unclear, our microarray (Supplemental Figure 3B) and qPCR data (Figure 6E) show a large overexpression (40-fold) of the WNT inhibitor Frzb/Sfrp3 following castration, which is reversed by DHT. This was consistent with previous data showing that $F r z b$ was one of the genes with the most sexually dimorphic expression pattern in mouse adrenals (46). This suggests that androgens could stimulate WNT signaling in the adrenal through repression of FrzB/SFRP3 expression.

Altogether, our data show that constitutive PKA activation in definitive steroidogenic cells accelerates $z G$-driven adrenal cortex renewal. This results in increased $z F$ differentiation (at the expense of $z G$ identity) and final conversion into a reticularis-like zone at the corticomedullary junction. This is correlated with increased recruitment and eventually exhaustion of both capsular and subcapsular progenitors. This phenomenon is counteracted in male adrenals by a positive effect of androgens on WNT signaling, which antagonizes PKA signaling and cortical cell turnover.

\section{Discussion}

The $\mathrm{zR}$ represents the innermost zone in the adrenal cortex whose development is required to initiate and support adrenarche, the prepubertal increase in adrenal androgen output that is thought to have evolved in human and great apes to promote brain maturation (reviewed in ref. 3). The cellular origin of the $\mathrm{zR}$ and the physiological mechanisms controlling its emergence and growth remain elusive. Because rats and mice lack postnatal expression of CYP17 in the adrenal, they have long been considered as nonrelevant experimental models to study adrenarche. However, the spiny mouse has been shown to express CYP17 and to produce adrenal-derived androgens from an individualized zR (47-49). This provides cues for possible usefulness of rodent models to explore underpinnings of adrenarche. Our data establish that constitutive PKA activation (driven by Prkarla genetic deletion) in the definitive cortex lineage of mice is a driving force to initiate and propagate the development of a reticularis-like zone, presumably through the conversion of the innermost fasciculata cells. This was demonstrated by morphological changes, acquisition of the necessary enzymatic machinery, and endocrine activity and raises the possibility that adrenarche in humans may result from activation of PKA signaling. A central role for PKA in this developmental process agrees with previous observations showing dexamethasone suppression of adrenal androgens (50-52) and a failure of children with ACTH receptor defects to experience adrenarche (53). Although ACTH/PKA signaling may be considered as an obligatory mediator of adrenarche, ACTH levels do not parallel rise in DHEA $(54,55)$. Despite numerous efforts, spread over decades, the existence of a specific hormonal inducer of adrenarche has not been identified (for review see refs. 3, 4, 56). Lack 
A

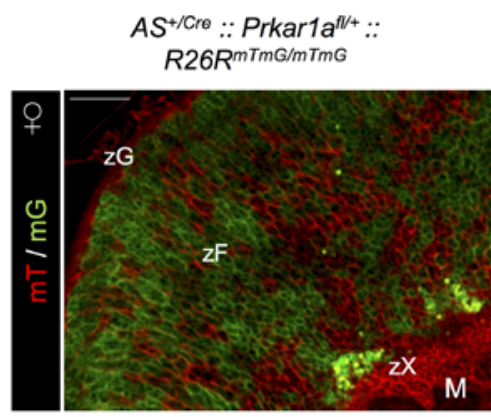

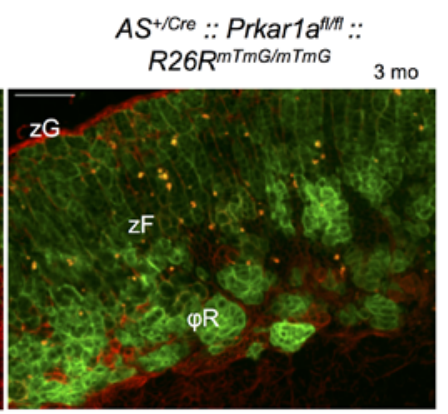

B

GFP (Recombination)

$\begin{array}{llll}4 & 5 & 10 & 13\end{array}$

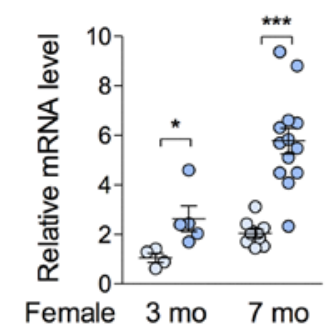

C $\operatorname{GFP}$ (Recombination)

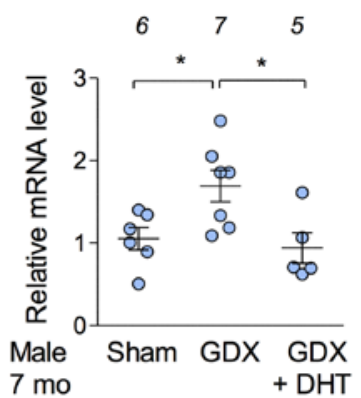

- $A S^{+/ C r e}::$ mTmG :: Prkar1a ${ }^{\text {fln }}$
D

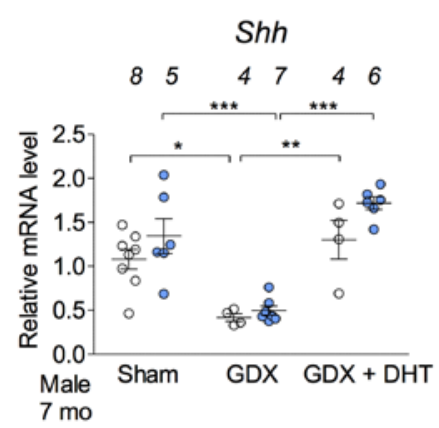

Progenitor markers

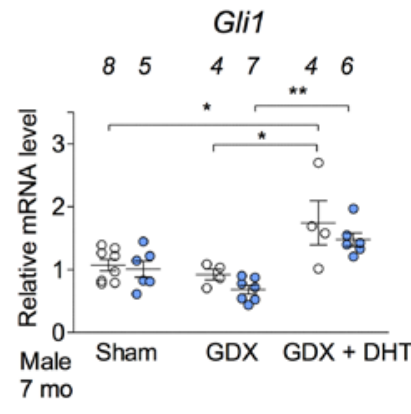

- $A S^{+/ C r e}:: m T m G$ :: Prkar1a ${ }^{f / /+}$

Ligand $\beta$-catenin signaling

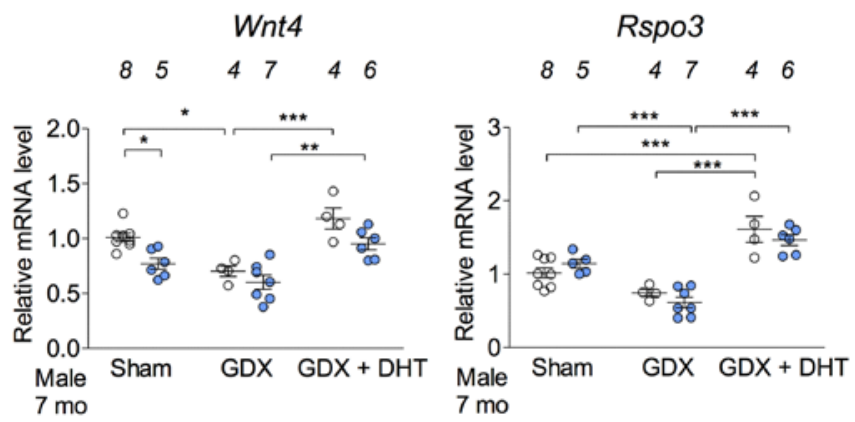

E

ßcatenin target genes

\section{$\underline{\text { Regulators of } \beta \text { catenin signaling }}$}

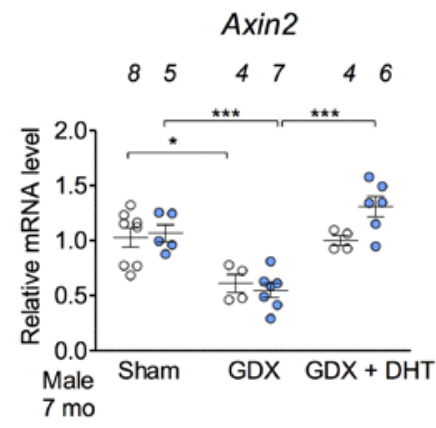

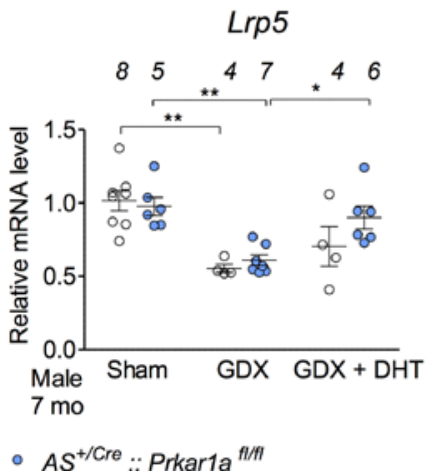

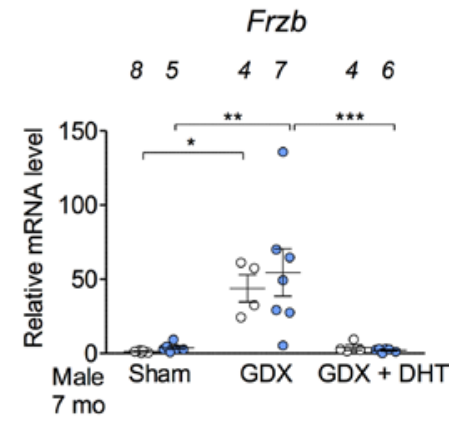

$7 \mathrm{mo}$

$\circ W T$

Figure 6. Constitutive activation of PKA signaling accelerates cortex renewal and stem/progenitor recruitment in an androgen-dependent manner. (A) Lineage-tracing studies showing change in centripetal cell turnover in adrenal cryosections from control (left) and DAdKO females (3 months). GFP and Tomato mark recombined and nonrecombined cells, respectively. Scale bars: $200 \mu \mathrm{m}$. (B) The rate of recombination in female adrenals was assessed by quantification of eGFP transcripts using qPCR. Lines in dot plots represent the mean relative quantification of eGFP expression (relative to the control counterparts) \pm SEM. Statistical analyses were conducted by Student's $t$ test. (C) The rate of recombination in male adrenals was assessed by quantification of eGFP transcripts using qPCR. Lines in dot plots represent the mean relative quantification of eGFP expression (relative to the control counterparts) \pm SEM. Statistical analyses were conducted by 1-way ANOVA followed by Bonferroni's test. ${ }^{* *} P<0.01$. (D and E) qPCR analyses of subcapsular (Shh)/ capsular (Gli1) progenitor markers, of ligand/receptor genes (Rspo3, Wnt4, Lrp5), WNT target genes (Axin2, Lef1), and Frzb WNT repressor. Lines in dot plots represent the mean quantification relative to WT 7-month-old mice of gene expression \pm SEM. Statistical analyses were conducted by 1-way ANOVA followed by Bonferroni's test. ${ }^{*} P<0.05,{ }^{* *} P<0.01,{ }^{* *} P<0.001$. The number of samples is indicated above dot plots.

of adrenarche in patients with an atrophic adrenal cortex lead to the hypothesis that adrenal growth mediated by trophic hormones, such as ACTH, should also be a determinant for the inner cortex to reach a critical size (depth) that in turn would trigger $\mathrm{zR}$ development $(53,57,58)$. Time lapse between fetal zone regression and $\mathrm{zR} /$ adrenarche onset may vary greatly among primates, from 6-8 years in humans to near synchronicity in the baboon (59). Interestingly, we observe a 1-year lag time between X-zone regression and pseudo-zR detection in DAdKO male mice (Figure $4 \mathrm{E}$ ) that is reduced to a nearly synchronous event in gonadectomized DAdKO males or in intact DAdKO females (Figure 2A and Figure 


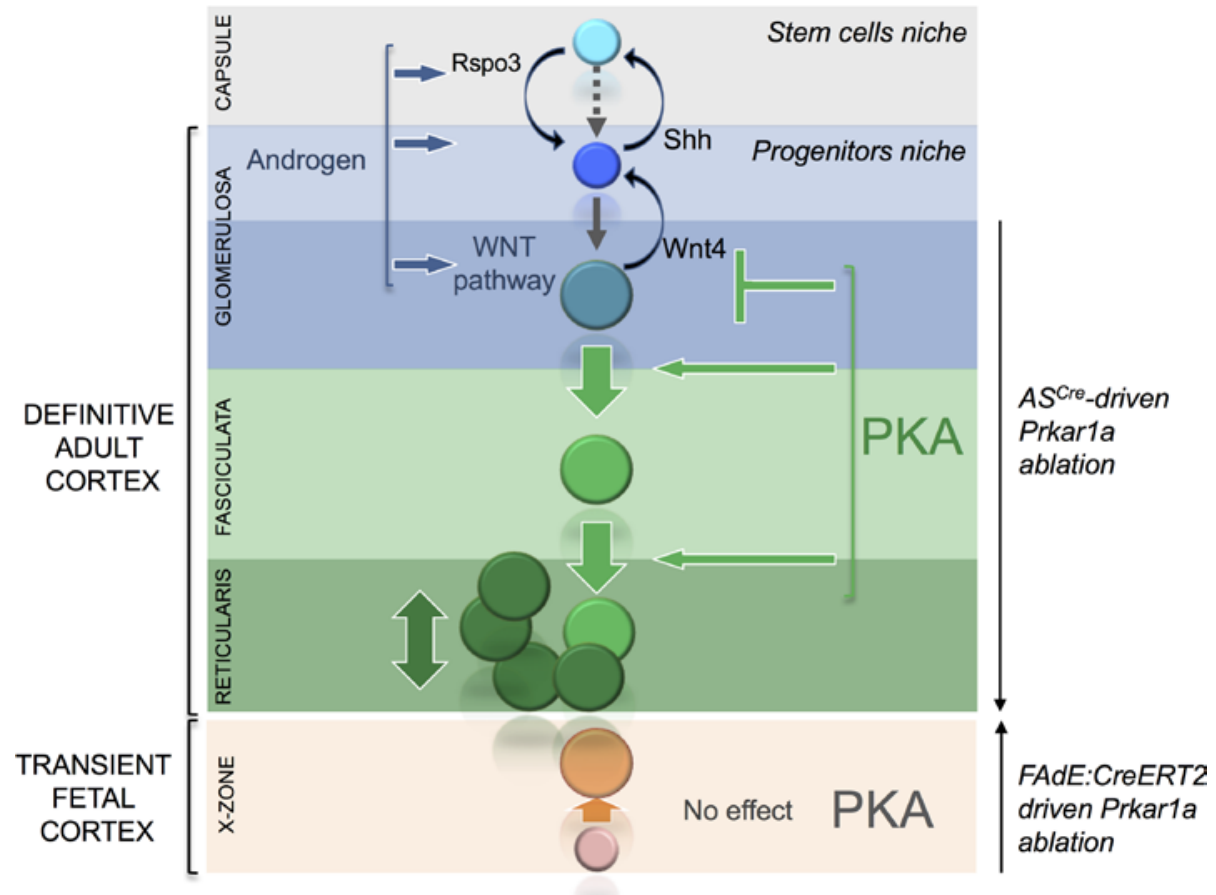

Figure 7. Model describing the role of PKA signaling in adrenal zonation, cortex replenishment, and interplay with testicular androgen. Solid gray arrow shows the main process of cell renewal occurring through recruitment of $S h h^{+}$subcapsular progenitors that differentiate as zona glomerulosa (zG) cells. Dashed gray arrow shows alternative renewal pathway relaying on the recruitment of capsular Rspo3/Gli ${ }^{+}$stem/progenitor cells that are essential to maintain Shh and Wht4 expression within subcapsular/zG cells and can, to a less extent, regenerate subcapsular progenitors. Large light green arrows symbolize the accelerated centripetal conversion of zG cells toward zona fasciculata (zF) identity and, subsequently, to cells with zona reticularis (zR) identity that eventually accumulate and expand (large dark green double-headed arrow) as cortex reaches critical size. Horizontal green bar-headed line and arrows show effects of constitutive PKA activation that represses $z G$ identity and promotes $z F$ and $z R$ conversion. Horizontal blue arrows show positive effects of testicular androgen on maintaining stem/progenitor pools, at least, through increased activation of WNT/ $\beta$-catenin signaling. Large orange arrow symbolizes the process of fetal X-zone formation occurring through recruitment of precursor cells maintaining FAdE active at E14.5, regardless of PKA activation.

5B). Our data demonstrate that the combined actions of unbuffered PKA signaling and a low androgenic milieu result in increased capsular/subcapsular progenitor recruitment, accelerated cell turnover, and, consequently, stimulation of cortical growth, potentially leading to a critical mass able to support the emergence of a zR (Figure 7). Eventually, this critical size triggers, by yet unknown sensing mechanisms, conversion of innermost $\mathrm{zF}$ cells into zR-like cells and growth of this new layer.

Beyond providing insights into the complex mechanisms that orchestrate adrenal zonation and zR onset, our data reveal clear sex differences in cortex remodeling capacity, with DAdKO female mice showing faster adult cortical cell renewal than DAdKO males. We show these differences have substantial consequences on adrenal disease susceptibility. Using lineage-tracing studies and hormonal challenge, we establish that resistance of males to develop cortical alteration and Cushing's syndrome following Prkarla genetic ablation relies on "protective" and reversible action of androgens competing with PKA signaling to slow down recruitment and centripetal differentiation of subcapsular/capsular progenitors into steroidogenic cells. By contrast, permissive (low-androgen) conditions encountered in females would favor PKA-prone cell turnover and cortex replenishment with mutant steroidogenic cells. The mechanism underlying androgen-dependent inhibition of PKA signaling remains to be elucidated but could involve the RSPO/WNT/ $\beta$-catenin pathway that is found substantially sustained under androgenic conditions in DAdKO males (Figure 6, D and E). Adrenocortical diseases occur generally earlier and are more frequent in women, with a sex ratio ranging from 1.5 to 3 for adrenal cancer $(60,61)$, Cushing's syndrome (62), or Carney complex nodular hyperplasia (63). Although sex steroids should be involved in this sex predilection, no supporting mechanisms have been identified so far. Based on our findings, we speculate that sex-related differences in the regulation of progenitor homeostasis, cortical cell turnover, and steroid biosynthetic pathways could account for the higher incidence and morbidity of adrenocortical lesions in women. 
In conclusion, we have identified PKA signaling as a central regulator of definitive inner cortex zonation, including conversion of $\mathrm{zF}$ into $\mathrm{zR}$, and thus as a possible mediator of adrenarche. Our study further identifies the progenitor compartment as an important target of PKA action promoting cortex renewal in a sex-dependent manner, in which androgens act as negative regulators. These findings could potentially lead to new insights to help explain the overrepresentation of adrenocortical diseases in women.

\section{Methods}

Mouse. The $A S^{+/ C r e}$ (11), $R 26 R^{m T m G}$ (64), Prkar1 $a^{f / / l}$ (65), and FAdE-Ad4BP-CreERT2 (FAdE-CreERT2) (10) mouse lines have been described previously. Mice were all maintained and bred on a mixed background. Throughout, WT refers to $A S^{+/ C r e}::$ Prkarla $^{+/ f l}:: R 26 R^{m T m G / m T m G}, A S^{+/+}::$Prkarla $^{+/ f l}:: R 26 R^{m T m G / m T m G}$, or $A S^{+/+}::$Prkarla $^{f l / f}:: R 26 R^{m T m G / m T m G}$. We did not observe any discernible difference among these genotypes (or with FAdE-CreERT2). For quantification of Prkarla transcripts, only $A S^{+/+}: \because$ Prkarla $^{+/ f t}:: R 26 R^{m T m G / m T m G}$ and

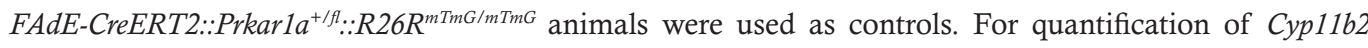
transcripts, only $A S^{+/ C r e}:: \operatorname{Prkarla}^{+/ f t}:: R 26 R^{m T m G / m T m G}$ animals were used as controls due to replacement of the Cyp11b2 gene by the Cre gene. Littermate control animals were used in all experiments. Cre activation during development was obtained by administration of a single dose of tamoxifen ( $3 \mathrm{mg}$ per $20 \mathrm{~g}$ of body weight by force feeding) in pregnant females carrying E14.5 embryos followed by 2 injections of $750 \mu \mathrm{g}$ progesterone (just after and 8 hours after force feeding).

Hormonal manipulations. Mice were injected subcutaneously with dexamethasone 21-acetate for 5 days in order to deplete endogenous ACTH production ( $75 \mu \mathrm{g}$ twice daily in corn oil). For androgen supplementation, 5.5-month-old male mice were injected subcutaneously with $5 \alpha$-androstan-17 $\beta$-ol-3-one for 5 weeks (75 $\mu$ g twice daily in corn oil).

Proliferation assays. Mice were injected i.p. with $50 \mathrm{mg} / \mathrm{kg}$ BrdU for 2 consecutive days and were sacrificed 1 day later.

Analysis of hormone levels. Blood samples were collected as previously described (19), and intra-adrenal steroids extraction was mentioned in ref. 18. Corticosterone and DHEA levels were determined by ELISA using commercially available kits (AR E-8100, LDN, and CAN-DH-490, Diagnostics Biochem). DHEAS levels were determined using RIA (66). ACTH levels were determined by multiplex assay (MPTMAG-49K, Merck Millipore). Aldosterone, cortisol, 21-deoxycortisol, and androstenedione levels were determined by LC-MS/MS (67). Renin activity rate in plasma was determined using a Fluorometric Sensolyte 520 Mouse Renin Assay Kit (AnaSpec Inc.).

Body composition analysis. Fat mass versus lean mass repartition of 7-month-old wild-type and mutant mice was determined using Echo Magnetic Resonance Imaging (QMR EchoMRI-900TM).

Insulin tolerance test. Insulin tolerance test was conducted on 4- to 6-month-old female mice fasted for 6 hours before intraperitoneal injection of $1 \mathrm{IU} / \mathrm{kg}$ of body weight of human insulin (Actrapid, Novo Nordisk) diluted in saline solution. Blood glucose concentration was determined using glucometer and Accu-Chek Active strips (Roche).

Immunohistological and Western blot analyses. Immunohistological and Western blot analysis methods have been previously described (18). See Supplemental Table 1 for conditions used.

Real-time PCR analysis. Total RNAs were extracted from 3-week-old to 14-month-old adrenal glands cleaned of adherent fat using the RNAII nucleotide extraction kit (Macherey Nagel) or the RNAqueous-Micro Kit (ThermoFisher Scientific), especially for 3-week-old adrenals, following the manufacturer's instructions. Primer pairs are listed in Supplemental Table 2.

Microarray analysis of gene expression. Adrenal gene expression profiles for four 7-month-old $A S^{+/ C r e}: \because P r-$ $k a r 1 a^{f l f l}$ and four WT littermates were analyzed using Affymetrix Mouse Gene 2.0 ST Arrays (raw and processed data are deposited on NCBI GEO platform [GSE103899]). Gene expression was normalized by RMA (Affy R package), and genotype comparisons were performed with the $t$ test. All $P$ values were adjusted by the Benjamini-Hochberg correction method. Heatmaps were generated with Excel and represent color-coded individual mean-centered gene expression levels (in the log2 space) in 4 WT and 4 knockout adrenals. Genes were ordered according to the mean fold change $(\log 2)$. The genes used in GSEA are listed in Supplemental Table 3.

Statistics. Statistical analyses were conducted by 2-tailed Student's $t$ test with Welch correction or 1-way ANOVA followed by Bonferroni's multiple comparison post-hoc test. $P$ values of less than 0.05 were considered significant. 
Study approval. All animal work was conducted according to French and European directives for the use and care of animals for research purposes and was approved by the local ethics committee, C2E2A (Comité d'Ethique pour 1'Expérimentation Animale en Auvergne), at Institut National de la Recherche Agronomique, research center Clermont-Theix-Lyon.

\section{Author contributions}

TD, ISB, AS, AMLM, PV, and AM conceived and designed the experiments; TD, ISB, AS, NM, IP, VD, and JCP performed the experiments; TD, ISB, and AM analyzed the data; FRB, MZ, KIM, and DTB contributed reagents/materials analysis tools; AM supervised the project, and TD and AM wrote the paper; and PV and DTB edited the paper.

\section{Acknowledgments}

We thank Y. Weinstein (Ben Gurion University of the Negev, Beer Sheva, Israel) for the gift of the 20aHSD antibody, A. Conley (University of California, Davis, California, USA) for the gift of CYP17 and CYB5B antibody, C. Gomez-Sanchez (University of Mississippi Medical Center, Jackson, Mississippi, USA) for the gift of CYP11B2 antibody, M. Thomas (Normandie Université, Rouen, France) for the gift of 3 $\beta$ HSD antibody, and H. Okamura (Kyoto University, Kyoto, Japan) for the gift of HSD3B6 antibody. We thank Y. Morel (Lyon, France) for giving us access to the hormonology service, for helpful discussion, and for critical reading of the manuscript. We thank A. Schedl (Nice, France) and E. Pignatti (Boston, MA) for critical reading of the manuscript. We also wish to thank Sandrine Plantade, Philippe Mazuel, and Khirredine Ouchen for caring for the transgenic mice. This work was funded through institutional support from CNRS, INSERM, Université Clermont Auvergne, and the French government IDEX-ISITE initiative 16-IDEX-0001 (CAP 20-25) as well as by grants from Région Auvergne-Rhône-Alpes (to TD), the Fondation Association pour la Recherche sur le Cancer (to TD), the Agence Nationale pour la Recherche (ANR14-CE12-000, to AM), and the NIH (R01 DK 084056, to DTB).

Address correspondence to: Antoine Martinez, Université Clermont Auvergne, GReD, CNRS UMR6293, INSERM U1103, Faculté de Médecine, CRBC, 28 place Henri Dunant, BP38, 63001 Clermont-Ferrand cedex, France. Phone: 33.4.73.40.74.09; Email: antoine.martinez@uca.fr.

1. Ishimoto H, Jaffe RB. Development and function of the human fetal adrenal cortex: a key component in the feto-placental unit. Endocr Rev. 2011;32(3):317-355.

2. Mesiano S, Jaffe RB. Developmental and functional biology of the primate fetal adrenal cortex. Endocr Rev. 1997;18(3):378-403.

3. Bird IM. In the zone: understanding zona reticularis function and its transformation by adrenarche. $J$ Endocrinol. 2012;214(2):109-111.

4. Rege J, Rainey WE. The steroid metabolome of adrenarche. J Endocrinol. 2012;214(2):133-143.

5. Brock BJ, Waterman MR. Biochemical differences between rat and human cytochrome P450c17 support the different steroidogenic needs of these two species. Biochemistry. 1999;38(5):1598-1606.

6. Keeney DS, Jenkins CM, Waterman MR. Developmentally regulated expression of adrenal 17 alpha-hydroxylase cytochrome P450 in the mouse embryo. Endocrinology. 1995;136(11):4872-4879.

7. Hershkovitz L, Beuschlein F, Klammer S, Krup M, Weinstein Y. Adrenal 20alpha-hydroxysteroid dehydrogenase in the mouse catabolizes progesterone and 11-deoxycorticosterone and is restricted to the X-zone. Endocrinology. 2007;148(3):976-988.

8. Holmes PV, Dickson AD. X-zone degeneration in the adrenal glands of adult and immature female mice. $J$ Anat. 1971;108(Pt 1):159-168.

9. Zubair M, Ishihara S, Oka S, Okumura K, Morohashi K. Two-step regulation of Ad4BP/SF-1 gene transcription during fetal adrenal development: initiation by a Hox-Pbx1-Prep1 complex and maintenance via autoregulation by Ad4BP/SF-1. Mol Cell Biol. 2006;26(11):4111-4121

10. Zubair M, Parker KL, Morohashi K. Developmental links between the fetal and adult zones of the adrenal cortex revealed by lineage tracing. Mol Cell Biol. 2008;28(23):7030-7040.

11. Freedman BD, et al. Adrenocortical zonation results from lineage conversion of differentiated zona glomerulosa cells. Dev Cell. 2013;26(6):666-673.

12. King P, Paul A, Laufer E. Shh signaling regulates adrenocortical development and identifies progenitors of steroidogenic lineages. Proc Natl Acad Sci USA. 2009;106(50):21185-21190.

13. Pignatti E, Leng S, Carlone DL, Breault DT. Regulation of zonation and homeostasis in the adrenal cortex. Mol Cell Endocrinol. 2017;441:146-155.

14. Vidal V, et al. The adrenal capsule is a signaling center controlling cell renewal and zonation through Rspo3. Genes Dev. 2016;30(12):1389-1394.

15. Berthon A, et al. Constitutive beta-catenin activation induces adrenal hyperplasia and promotes adrenal cancer development. Hum Mol Genet. 2010;19(8):1561-1576. 
16. Berthon A, et al. WNT/ $\beta$-catenin signalling is activated in aldosterone-producing adenomas and controls aldosterone production. Hum Mol Genet. 2014;23(4):889-905.

17. Walczak EM, et al. Wnt signaling inhibits adrenal steroidogenesis by cell-autonomous and non-cell-autonomous mechanisms Mol Endocrinol. 2014;28(9):1471-1486.

18. Drelon C, et al. PKA inhibits WNT signalling in adrenal cortex zonation and prevents malignant tumour development. Nat Commun. 2016;7:12751.

19. Sahut-Barnola I, et al. Cushing's syndrome and fetal features resurgence in adrenal cortex-specific Prkarla knockout mice. PLoS Genet. 2010;6(6):e1000980.

20. Chida D, et al. Melanocortin 2 receptor is required for adrenal gland development, steroidogenesis, and neonatal gluconeogenesis. Proc Natl Acad Sci USA. 2007;104(46):18205-18210.

21. Clark AJ, Weber A. Molecular insights into inherited ACTH resistance syndromes. Trends Endocrinol Metab. 1994;5(5):209-214.

22. Metherell LA, et al. Mutations in MRAP, encoding a new interacting partner of the ACTH receptor, cause familial glucocorticoid deficiency type 2. Nat Genet. 2005;37(2):166-170.

23. Kirschner LS, et al. Mutations of the gene encoding the protein kinase A type I-alpha regulatory subunit in patients with the Carney complex. Nat Genet. 2000;26(1):89-92.

24. Lambert-Langlais S, et al. A transgenic mouse line with specific Cre recombinase expression in the adrenal cortex. Mol Cell Endocrinol. 2009;300(1-2):197-204.

25. Pihlajoki M, et al. Conditional mutagenesis of Gata6 in SF1-positive cells causes gonadal-like differentiation in the adrenal cortex of mice. Endocrinology. 2013;154(5):1754-1767.

26. Mueller JW, Gilligan LC, Idkowiak J, Arlt W, Foster PA. The regulation of steroid action by sulfation and desulfation. Endocr Rev. 2015;36(5):526-563.

27. Endoh A, Kristiansen SB, Casson PR, Buster JE, Hornsby PJ. The zona reticularis is the site of biosynthesis of dehydroepiandrosterone and dehydroepiandrosterone sulfate in the adult human adrenal cortex resulting from its low expression of 3 beta-hydroxysteroid dehydrogenase. J Clin Endocrinol Metab. 1996;81(10):3558-3565.

28. Gell JS, et al. Adrenarche results from development of a 3beta-hydroxysteroid dehydrogenase-deficient adrenal reticularis. $J$ Clin Endocrinol Metab. 1998;83(10):3695-3701.

29. Miller WL. Early steps in androgen biosynthesis: from cholesterol to DHEA. Baillieres Clin Endocrinol Metab. 1998;12(1):67-81.

30. Nakamura Y, et al. Type 5 17beta-hydroxysteroid dehydrogenase (AKR1C3) contributes to testosterone production in the adrenal reticularis. J Clin Endocrinol Metab. 2009;94(6):2192-2198.

31. Rege J, Nakamura Y, Wang T, Merchen TD, Sasano H, Rainey WE. Transcriptome profiling reveals differentially expressed transcripts between the human adrenal zona fasciculata and zona reticularis. J Clin Endocrinol Metab. 2014;99(3):E518-E527.

32. Suzuki T, et al. Developmental changes in steroidogenic enzymes in human postnatal adrenal cortex: immunohistochemical studies. Clin Endocrinol (Oxf). 2000;53(6):739-747.

33. Kraemer FB. Adrenal cholesterol utilization. Mol Cell Endocrinol. 2007;265-266:42-45.

34. Lefrançois-Martinez AM, et al. Product of side-chain cleavage of cholesterol, isocaproaldehyde, is an endogenous specific substrate of mouse vas deferens protein, an aldose reductase-like protein in adrenocortical cells. J Biol Chem. 1999;274(46):32875-32880.

35. El Ghorayeb N, Bourdeau I, Lacroix A. Role of ACTH and other hormones in the regulation of aldosterone production in primary aldosteronism. Front Endocrinol (Lausanne). 2016;7:72.

36. Funder JW. The potential of ACTH in the genesis of primary aldosteronism. Front Endocrinol (Lausanne). $2016 ; 7: 40$.

37. Arnaldi G, et al. Diagnosis and complications of Cushing's syndrome: a consensus statement. J Clin Endocrinol Metab. 2003;88(12):5593-5602.

38. Arnaldi G, Scandali VM, Trementino L, Cardinaletti M, Appolloni G, Boscaro M. Pathophysiology of dyslipidemia in Cushing's syndrome. Neuroendocrinology. 2010;92 Suppl 1:86-90.

39. Jopek K, et al. Transcriptome profile of rat adrenal evoked by gonadectomy and testosterone or estradiol replacement. Front Endocrinol (Lausanne). 2017;8:26.

40. Walczak EM, Hammer GD. Regulation of the adrenocortical stem cell niche: implications for disease. Nat Rev Endocrinol. 2015;11(1):14-28

41. Bandiera R, et al. WT1 maintains adrenal-gonadal primordium identity and marks a population of AGP-like progenitors within the adrenal gland. Dev Cell. 2013;27(1):5-18.

42. Guasti L, et al. Dlk1 up-regulates Gli1 expression in male rat adrenal capsule cells through the activation of $\beta 1$ integrin and ERK1/2. Endocrinology. 2013;154(12):4675-4684.

43. Wood MA, et al. Fetal adrenal capsular cells serve as progenitor cells for steroidogenic and stromal adrenocortical cell lineages in M. musculus. Development. 2013;140(22):4522-4532.

44. Lee SH, et al. Androgen signaling is a confounding factor for $\beta$-catenin-mediated prostate tumorigenesis. Oncogene 2016;35(6):702-714

45. Schweizer L, et al. The androgen receptor can signal through Wnt/beta-Catenin in prostate cancer cells as an adaptation mechanism to castration levels of androgens. BMC Cell Biol. 2008;9:4.

46. El Wakil A, Mari B, Barhanin J, Lalli E. Genomic analysis of sexual dimorphism of gene expression in the mouse adrenal gland. Horm Metab Res. 2013;45(12):870-873.

47. Quinn TA, et al. Ontogeny of the adrenal gland in the spiny mouse, with particular reference to production of the steroids cortisol and dehydroepiandrosterone. Endocrinology. 2013;154(3):1190-1201.

48. Quinn TA, Ratnayake U, Castillo-Melendez M, Moritz KM, Dickinson H, Walker DW. Adrenal steroidogenesis following prenatal dexamethasone exposure in the spiny mouse. J Endocrinol. 2014;221(2):347-362.

49. Quinn TA, Ratnayake U, Dickinson H, Castillo-Melendez M, Walker DW. The feto-placental unit, and potential roles of dehydroepiandrosterone (DHEA) in prenatal and postnatal brain development: A re-examination using the spiny mouse. $J$ Steroid Biochem Mol Biol. 2016;160:204-213.

50. Abraham GE. Ovarian and adrenal contribution to peripheral androgens during the menstrual cycle. J Clin Endocrinol Metab. $1974 ; 39(2): 340-346$ 
51. Kim MH, Hosseinian AH, Dupon C. Plasma levels of estrogens, androgens and progesterone during normal and dexamethasone-treated cycles. J Clin Endocrinol Metab. 1974;39(4):706-712.

52. Rich BH, Rosenfield RL, Lucky AW, Helke JC, Otto P. Adrenarche: changing adrenal response to adrenocorticotropin. J Clin Endocrinol Metab. 1981;52(6):1129-1136.

53. Weber A, Clark AJ, Perry LA, Honour JW, Savage MO. Diminished adrenal androgen secretion in familial glucocorticoid deficiency implicates a significant role for ACTH in the induction of adrenarche. Clin Endocrinol (Oxf). 1997;46(4):431-437.

54. Mellon SH, Shively JE, Miller WL. Human proopiomelanocortin-(79-96), a proposed androgen stimulatory hormone, does not affect steroidogenesis in cultured human fetal adrenal cells. J Clin Endocrinol Metab. 1991;72(1):19-22.

55. Penhoat A, Sanchez P, Jaillard C, Langlois D, Bégeot M, Saez JM. Human proopiomelanocortin-(79-96), a proposed cortical androgen-stimulating hormone, does not affect steroidogenesis in cultured human adult adrenal cells. J Clin Endocrinol Metab. 1991;72(1):23-26

56. Hornsby PJ. Adrenarche: a cell biological perspective. J Endocrinol. 2012;214(2):113-119.

57. Urban MD, Lee PA, Gutai JP, Migeon CJ. Androgens in pubertal males with Addison's disease. J Clin Endocrinol Metab. 1980;51(4):925-929.

58. Brunelli VL, Chiumello G, David M, Forest MG. Adrenarche does not occur in treated patients with congenital adrenal hyper plasia resulting from 21-hydroxylase deficiency. Clin Endocrinol (Oxf). 1995;42(5):461-466.

59. Ducsay CA, Hess DL, McClellan MC, Novy MJ. Endocrine and morphological maturation of the fetal and neonatal adrenal cortex in baboons. J Clin Endocrinol Metab. 1991;73(2):385-395.

60. Fassnacht M, Libé R, Kroiss M, Allolio B. Adrenocortical carcinoma: a clinician's update. Nat Rev Endocrinol. 2011;7(6):323-335.

61. Abiven-Lepage G, et al. Adrenocortical carcinoma and pregnancy: clinical and biological features and prognosis. Eur J Endocrinol. 2010;163(5):793-800.

62. Steffensen C, Bak AM, Rubeck KZ, Jørgensen JO. Epidemiology of Cushing's syndrome. Neuroendocrinology. 2010;92 Suppl 1:1-5.

63. Bertherat J, et al. Mutations in regulatory subunit type $1 \mathrm{~A}$ of cyclic adenosine 5'-monophosphate-dependent protein kinase (PRKAR1A): phenotype analysis in 353 patients and 80 different genotypes. J Clin Endocrinol Metab. 2009;94(6):2085-2091.

64. Muzumdar MD, Tasic B, Miyamichi K, Li L, Luo L. A global double-fluorescent Cre reporter mouse. Genesis. 2007;45(9):593-605

65. Kirschner LS, et al. A mouse model for the Carney complex tumor syndrome develops neoplasia in cyclic AMP-responsive tissues. Cancer Res. 2005;65(11):4506-4514.

66. de Peretti E, Forest MG. Pattern of plasma dehydroepiandrosterone sulfate levels in humans from birth to adulthood: evidence for testicular production. J Clin Endocrinol Metab. 1978;47(3):572-577.

67. Meunier C, et al. Development and validation of a method using supported liquid extraction for aldosterone determination in human plasma by LC-MS/MS. Clin Chim Acta. 2015;447:8-15. 